\title{
An Evaluation of Pooling Strategies for RT-qPCR Testing for SARS-CoV-2 Infection: A Pragmatic Multi-site Parallel Operational Study
}

Raymundo Lo, ${ }^{1}$ Agnes Barrientos, ${ }^{2}$ Bernadette Espiritu, ${ }^{3}$ Farrah Kristine Santiago, ${ }^{4}$ Amado Tandoc III, ${ }^{5}$ Januario Veloso, ${ }^{6}$ Soccoro Yañez ${ }^{7}$

${ }^{1}$ COVID-19 Testing Laboratory, Philippine Children's Medical Center, Quezon City

${ }^{2}$ Department of Pathology and Laboratory Services, University of Perpetual Help System DALTA Medical Center, Las Piñas, Philippines

${ }^{3}$ Bulacan Medical Center, Philippines

${ }^{4}$ Section of Hematology, Philippine Children's Medical Center Pathology Division, Quezon City

${ }^{5}$ Laboratory Research Division, Research Institute for Tropical Medicine, Alabang, Muntinlupa, Philippines

${ }^{6}$ Medical Research Laboratory, University of the Philippines-Philippine General Hospital Medical Center, Ermita, Manila

${ }^{7}$ Section of Anatomic Pathology, University of the East Ramon, Magsaysay Memorial Medical Center, Quezon City, Philippines

\section{ABSTRACT}

Background and Objectives. Sample pooling of COVID-19 PCR tests has been recently proposed as a lowcost alternative to individual tests. This multi-site, laboratory-based, proof-of-concept study explores the feasibility of pooled SARS-COV-2 RT-aPCR testing, by demonstrating the effect of pooling on sensitivity, specificity, accuracy, number of tests saved, and turnaround time.

Methodology. The research was conducted in two experiments. In Experiment 1, archival nasopharyngeal (NPS) and oropharyngeal (OPS) swab samples were diluted to simulate 5, 10, and 20 sized pools, and tested for SARS-CoV-2 RNA using RT-qPCR. In Experiment 2, actual nasopharyngeal and oropharyngeal swab samples were collected from asymptomatic low-risk volunteers. Aliquots of the samples were pooled following the 5, 10-5, and 20-10-5 multi-staged Dorfman pooling methods and tested. The sensitivity, specificity, accuracy, test savings, and turnaround time for each pooling method were documented.

Results and Conclusions. The study provided evidence that pooling of NP and OP samples for SARS-COV-2 RNA detection using RT-qPCR is feasible and can be implemented in the Philippines. A 2-stage Dorfman 5 pooling strategy appears to be the best method, because it has the highest over-all accuracy, while still achieving acceptable test savings, and turnaround time. Pooling of nasopharyngeal and oropharyngeal swab samples prior to RT-qPCR testing may be considered by select molecular diagnostic laboratories to further increase testing capacity and at the same time reduce the cost of testing.

Key words: pooled testing, specimen pooling, RT-qPCR, COVID-19, SARS-CoV-2

ISSN 2507-8364 (Online)

Printed in the Philippines.

Copyright $(2020$ by the PJP.

Received: 11 December 2020

Accepted: 20 December 2020.

Published online first: 6 January 2021.

https://doi.org/10.21141/PJP.2020.12

Corresponding author: Farrah Kristine F. Santiago, MD, FPSP E-mail:fqfsantiago@gmail.com

\section{INTRODUCTION}

The capacity of the Philippine healthcare system to perform NAATs for detecting SARS-CoV-2 nucleic acids was extremely limited at the beginning of the pandemic. ${ }^{1}$ Testing capacity steadily increased with the certification and licensing of new COVID-19 diagnostic molecular laboratories, now reaching to about 100. As of August 2020, the testing capacity of the whole country is about 27,800 tests per day. ${ }^{2}$ The WHO has suggested around $10-30$ tests per confirmed case as a general benchmark of adequate testing. ${ }^{3}$ In August 2020, the Philippines did 8.2 tests per positive case ${ }^{4}$ which placed the country in the "moderate" category. According to OurWorldInData.org, as of September 30, 2020, the Philippines did 12.8 tests per new confirmed case, with a daily new case positive rate of nearly 3,000 for the week of September 26 to October 3, 2020. ${ }^{3}$ Based on the above recommendations, the Philippines should be doing 30,000 to 90,000 tests daily. The cost of testing, however, remains a challenge.

The Philippine government gradually transitioned from enhanced community quarantine to more relaxed quarantine regimes. ${ }^{5}$ Potential challenges that came with 
this reopening of social activities include (1) possible surges in the number of new infections, (2) the need for certain groups of workers to be tested on a regular basis, and (3) the need for patient testing to prioritize access to invasive and surgical procedures.

At present, the gold standard for the diagnosis of COVID-19, is by nucleic acid amplification testing (NAAT), which includes quantitative reverse transcriptase polymerase chain reaction (RT-qPCR). In the case of COVID-19, NAATs amplify a tiny amount of viral genetic material using a nasopharyngeal or oropharyngeal swab sample. The demand for this type of SARS-CoV-2 testing has drastically increased in many health care systems, and resulted in shortages of reagents and materials to conduct the test, or exceeded the capacity limits of the testing laboratories.

Reverse transcriptase polymerase chain reaction (RTPCR) is a laboratory technique that combines reverse transcription of RNA into DNA and amplification of specific DNA targets. The chain reaction relies on small DNA sequence primers that are designed to specifically recognize complementary sequences on the RNA viral genome and the reverse transcriptase to generate a short complementary DNA copy (cDNA) of the viral RNA (called amplicons). In real-time or quantitative PCR (RT-qPCR), the amplification of DNA is monitored in real time as the PCR reaction progresses. Detection of the viral copies is achieved using a fluorescent dye or DNA probe labeled with a fluorescent molecule and a quencher molecule, as in the case of TaqMan assays. An automated system then repeats the amplification process for about 40 cycles until the viral cDNA can be detected.

The Philippine Society of Pathologists Inc. (PSP), in its position paper of May 29, 2020, recommended the implementation of pooled RT-PCR COVID testing to expand testing capacity, reduce turnaround time and conserve reagents and human resources. ${ }^{6}$

Incorporating specimen pooling strategies into RTqPCR testing may be a viable solution to facilitate the calibrated exit of the country from community quarantine to a more relaxed economic and social activity. Pooling methods entail getting aliquots from several samples and combining them together in a single tube for RT-PCR testing, reducing the number of actual tests performed.

However, several questions about sample pooling remain. For instance, the largest pooling size, $n$, such that the sensitivity of detecting low copy numbers of nucleic acids would still be acceptable (sensitivity: 90\%) has not yet been established in the local setting. The largest pooling size, $n$, is the maximum pooling size that can be allowed in a clinical laboratory such that the RT-qPCR would still have a sensitivity of $90 \%$, a performance characteristic the PSP considers as the minimum acceptable sensitivity for clinical testing.

The effect of pooling on the analytic sensitivity also needs to be studied by looking at how much the Ct-values will change between the original specimen and the pooled (diluted) specimen. Pool sizes with the least change in Ctvalues can be considered acceptable.

Prevalence rates are critical in establishing pooling strategies. Unfortunately, with the limited testing done mainly on symptomatic cases, there are no reliable local prevalence data in the general asymptomatic and low risk population. For the purposes of this study, however, an arbitrary but reasonable prevalence rate of $5 \%$ is used as a basis to inform the design of pooling methods. Pooling sizes of $n=5, n=10$, and $n=20$ are the pool sizes likely to be optimal for disease prevalence ranging from $0.3 \%$ to $20 \%^{7,8}$ and are the pooling sizes that were included in this study.

Several groups, including local scientists, have recommended optimal pooling strategies and sizes by maximizing the expected savings on the number of tests needed using computer simulations. ${ }^{7-24}$

Some of these included feasibility and proof-of-concept studies using actual samples. A summary of the studies and papers are presented in Table 1. A review of their findings is discussed in the succeeding paragraphs.

Well-described and popular pooling methods include the Dorfman, which includes the two-stage hierarchical (D2) and three-stage hierarchical (D3) variations, the repeated sub-pooling method of Sterrett, the halving method by

\begin{tabular}{lll}
\hline \multicolumn{2}{l}{ Table 1. Summary of pooling studies reviewed } & Study site \\
\hline Authors & Type of Pooling Study & Nebraska, USA \\
\hline (Abdalhamid et al., 2020) & Computer Simulations and testing with actual specimens & Nebraska, USA \\
& Computer simulations & Manila, Philippines \\
& Computer simulations & Germany \\
\hline (Bilder \& Tebbs, 2012) & Computer simulations & Japan \\
& Actual specimens & California, USA \\
\hline (Caoili et al., 2020) & Actual specimens & MA, USA \\
& Mathematical theoretical discussion & Germany \\
\hline (Deckert et al., 2020) & Actual specimens & Utah, USA \\
\hline (Hirotsu et al., 2020) & Mathematical theoretical discussions & Australia \\
\hline (Hogan et al., 2020) & Mathematical theoretical discussions & Jerusalem, Israel \\
& Computer Simulations & Israel \\
\hline (Litvak et al., 1994) & Computer simulations and testing with actual specimens (used automated machines) & USA \\
\hline (Lohse et al., 2020) & Actual specimens & Spain \\
\hline & Actual specimens & USA \\
\hline
\end{tabular}


Litvak et al., and the array or matrix based methods by Phatarford and Sudbury.

In the Dorfman method, the individual samples are $1^{\text {st }}$ pooled in $\mathrm{n}$ samples and tested. If the master pool is negative, all $\mathrm{n}$ samples are classified as negative. If the master pool tests positive, individual testing of each sample is done, and the samples are classified accordingly. ${ }^{25}$

In the repeated sub-pooling method by Sterrett, the strategy starts like the Dorfman methods, but samples included in pools that test positive are tested randomly, rather than exhaustively, until the $1^{\text {st }}$ positive individual sample is found. Once the first positive is found, the rest of the samples that have not yet tested are re-pooled and retested. If the pool tests negative, all other samples in this pool are classified as negative and testing is done on the other pools. The process is repeated until all positives are identified. Sterrett was able to show that the testing efficiency can be increased from 0.8 to 0.86 , for example, in a test population with prevalence of $0.01 .{ }^{20}$

The "halving method" proposed by the group of Litvak, splits the samples into two or more sub pools. Further splitting or individual testing can be done on each sub pool that tested positive. All samples in pools that test negative are classified as negative. In this method, each successive split creates two new equally sized sub pools. In real applications, however, the method only involves 3 to 4 levels of sub-pooling. Litvak and his team compared their method with 4 different pooling strategies, including variations of the Dorfman, and further variations of their halving technique which adjusts the number of times positive and negative pools are tested before assigning a classification. In their mathematical discussion, they found that all pooling strategies they tested resulted in cost savings with differences in false negatives and false positives and that the final choice should take into consideration the consequences of these errors in the actual setting. ${ }^{16}$

Square or rectangular matrix or array based pooling methods, with popular designs by Phatarford and Sudbury, are usually used with high throughput screening platforms. In these methods, $n \times n$ or $n \times m$ matrix-like grids of specimens are created. Each $n$ rows and $m$ columns are tested as a pool. Each pool can be tested twice to increase sensitivity. Samples that lie at the intersection of positive rows and positive columns are tested individually to decode the positives from the negatives. Specimens lying outside of these intersecting rows and columns are declared negative. Phatarford and his colleagues demonstrated mathematically that placing the samples in a square array and pooling rows and columns has substantial advantages, particularly in the reduction of false negatives. ${ }^{17}$ They compared the simple Dorfman, to Halving strategy by Fincuan, and the repeated pooling by Sterret. ${ }^{18,25}$ The number of tests saved, measured by the number of tests/person as well as the probability of false negative samples were calculated and compared between the Dorfman and the array methods. They were able to mathematically demonstrate the superiority of the array methods compared to the simple Dorfman procedures. ${ }^{18,25}$
SUMMARY OF LITERATURE ON POOLING METHODS ON SIMULATED OR ACTUAL SAMPLES

The group of Hirotsu in Japan validated the feasibility of pooling samples using serial dilution analysis and spike-in experiment using synthetic DNA and nucleic acids extracted from SARS-CoV-2 positive and negative patients. They also studied a total of 1,000 individuals, 667 of which are 'healthy' (195 healthcare workers and 472 hospitalized patients with other disorders than COVID-19 infection) individuals and 333 are infectionsuspected patients with cough and fever. Their serial dilution analysis showed a limit of detection of around 10100 copies. Their spike-in experiment demonstrated that RT-qPCR can detect positive signal in pooling samples of SARS-CoV-2 negative and positive patient at the 5-, 10-, 20-fold dilution. They performed screening using their pooling strategy during the months up to April 2020, and they were able to identify 12 COVID-19 patients in 333 suspected patients (3.6\%) and zero in 667 'healthy', using only a total of 538 tests instead of the 1000 which would have been required if done without pooling. ${ }^{12}$

Hogan and colleagues used a simple 2-stage Dorfman pooling strategy using 9 or 10 samples per pool to test and screen for SARS-CoV-2. They were able to screen 292 pools, corresponding to $2740 \mathrm{NP}$ samples and 148 bronchoalveolar lavage samples. They reported only 1 false-positive reading and an expected slight loss in sensitivity. ${ }^{13}$

Perchetti et al., noted a 2 Ct value loss in analytical sensitivity with 1:4 pooling of samples using CDC-based RT-PCR laboratory developed assay. ${ }^{14}$

Mulu et al., demonstrated differences in Ct values in experimental pools of 2 to $10 .{ }^{15}$ The group of Lohse evaluated Simple Dorfman pooling methods of varying pool sizes on actual SARS-CoV-2 samples. They were able to show that the difference between the Ct values of pooled and non-pooled specimens ranged up to 5 points. Based on their data, they were able to analyze 1161 samples using only 267 tests to detect 23 positives resulting in large cost savings. Their data suggests that pooling of up to 30 samples per pool can be used but they caution against the possibility of decreased sensitivity in patients 14-21 days after symptomatic infection. ${ }^{24}$

Noriega and his group discussed the applicability of a pooled-sample testing protocol to screen large populations more rapidly and with limited resources using a Bayesian inference analysis. Hierarchical testing stages were implemented, and their sensitivities were benchmarked against early COVID-19 testing data. They calculated the optimal pool size, increases in throughput and case detection abilities as a function of disease prevalence. They concluded that even for moderate losses in test sensitivity due to pooling, substantial increases in testing throughput and detection efficiency can be expected. ${ }^{17}$

Shani Narkiss and colleagues discussed two possible optimized pooling strategies for diagnostic SARSCoV-2 testing on a large scale. The first uses a simple informationtheoretic heuristic to derive a highly efficient re-pooling 
protocol where an estimate of the target frequency determines the initial pool size and any subsequent pools found positive are re-pooled at half-size and tested again. This was found to reduce the number of tests required dramatically, when the prevalence is less than $5 \%$. The second method is simpler and uses an optimized one-time pooling followed by individual tests on positive pools. They were able to show that this approach is just as efficient for prevalence ranging from $5 \%$ to less than $20 \%$. Compared to naive individual testing and alternative matrix methods, they show that their methods can be practical. ${ }^{8}$

Shental and colleagues developed P-BEST - a method for Pooling-Based Efficient SARS-CoV-2 Testing, using a non-adaptive group-testing approach, which significantly reduces the number of tests required to identify all positive subjects within a large set of samples. This method tests samples by pooling into groups. Each sample, however, is part of multiple pools and uses a combinatorial pooling strategy based on compressed sensing method. They evaluated this P-BEST strategy using leftover samples in a proof-of-concept study. They pooled 384 patient samples into 48 pools. Five sets of 384 samples, containing 1-5 positive carriers were tested using the method and all positive carriers in each set were correctly identified. ${ }^{19}$

Torres and colleagues conducted a proof-of-concept study and a mini trial where they evaluated the efficacy of a pooling strategy in Covid-19 testing. They used a total of 20 mini-pools containing either $5(n=10)$ or $10(n=10)$ nasopharyngeal exudates collected in universal transport medium, each of which included a unique positive NP specimen. ${ }^{21}$

\section{OBJECTIVES}

The general objective of this 2-part study is to determine the effect of nasopharyngeal and oropharyngeal swab sample pooling on the test sensitivity, number of tests saved and turnaround time of RT-qPCR testing for SARS-CoV-2 RNA.

Specifically, this study aims to determine which of the pooling sizes $(n=5, n=10$, and $n=20)$ would retain an acceptable test sensitivity $(90 \%)$ in identifying samples with low copies of viral RNAs (archival samples with Ct values ranging from 30-38). Pooling strategies are being used routinely in nucleic acid amplification for transfusiontransmissible infections in blood banking. ${ }^{26,27,28}$

This study also specifically aims to compare variations of the Dorfman pooling strategy (2-stage Dorfman 5, 3-stage Dorfman 10-5, and 4-stage Dorfman 20-10-5) to no-pooling and determine test accuracy, test savings and turnaround time.

\section{METHODOLOGY}

This study conducted laboratory-based parallel multi-site operational pragmatic experiments using a combination of archival and actual patient samples in 2 phases. Experiment 1 was designed to answer specific objective 1 and was conducted at the Research Institute for Tropical Medicine (RITM). Experiment 2, which was designed to answer specific objective 2, was conducted at the Philippine Children's Medical Center (PCMC) and University of Perpetual Help Dalta Medical Center (UPHDMC). The study protocols, including the informed consent forms, were reviewed and approved by the institutional review boards of RITM (RITM IRB 2020-022), PCMC (PCMC IR-EC 2020-046) and UPHDMC (UPHS-IERB 2020-003).

\section{Interpretation of pooled sample results (Experiment 1)}

For Experiment 1, nasopharyngeal and oropharyngeal specimens that have been previously collected and tested in RITM, with Ct values ranging between 30 to 38 were identified and retrieved by convenience sampling. These specimens have been properly stored at $2{ }^{\circ} \mathrm{C}$ to $8{ }^{\circ} \mathrm{C}$ for no more than 72 hours. Specimens that were not tested within 72 hours were stored at $-80^{\circ} \mathrm{C}$.

In Experiment 1, we performed an experiment similar to the method used by Abdalhamid et al (2020). ${ }^{9}$ Previously characterized positive nasopharyngeal + oropharyngeal $(\mathrm{NP}+\mathrm{OP})$ swab specimens with high Ct-values (Ct-value $>30<38$ ) were identified. The Ct-values obtained from previous testing were considered as an indirect measure of the specimen's actual starting nucleic acid copies, with Ct values higher than 30 taken generally to mean low nucleic acid copies. The samples were selected based on the results of their initial real-time RT-PCR runs as well as the quality and remaining volume of the original samples. Undiluted samples were tested along with the diluted samples to ensure that same testing conditions were met for both undiluted and diluted samples.

Fifty (50) uL aliquots from each sample were diluted (as described below) to simulate the different pool sizes at the worst possible pooling scenarios - where only 1 specimen is positive out of the pool:

1. For pool size $\mathrm{n}=5,50 \mathrm{uL}$ of specimen were added to $200 \mathrm{uL}$ of diluent/buffer

2. For pool size $\mathrm{n}=10,50 \mathrm{uL}$ of specimen were added to $450 \mathrm{uL}$ of diluent/buffer

3. For pool size $\mathrm{n}=20,50 \mathrm{uL}$ of specimen were added to $950 \mathrm{uL}$ of diluent/buffer

The diluted samples underwent nucleic acid extraction and RT-qPCR using Qiagen Viral RNA Kit and Maccura SARSCoV-2 Fluorescent PCR Kit, respectively. ABI 7500 Fast Real-Time PCR was used for real-time PCR amplification of the PCR reaction mix. All procedures were performed strictly according to the manufacturer's instructions for use and followed strict biosafety guidelines and good clinical laboratory practices. Results were recorded and encoded in electronic data collection forms.

For Experiment 2, volunteer employees from a local supermarket chain were interviewed and invited to participate in the study. Supermarket employees were selected for the pooling population as proposed by the Philippine Society of Pathologists Inc.'s position paper entitled "Diagnostic Testing Strategy to Manage COVID-19 Pandemic." ${ }^{6}$ In the position paper, an expanded targeted testing for asymptomatic population was listed, including testing employees who are at risk due to higher exposures and contact. 
They were selected from a list of potential participants that were provided by the study sponsors. All of them were asymptomatic and were classified under "sub-groups D (patients and healthcare workers with no symptoms but relevant history of travel and/or contact)" as defined in the DOH Department Memorandum 2020-0258: Updated Interim Guidelines on Expanded Testing for COVID-19. ${ }^{29}$ Three store locations were pre-selected by the study sponsor: Branch 1: 120 workers, Branch 2: 250 workers, and Branch 3: 80 workers.

Employees who had symptoms of fever, cough, colds, or shortness of breath at the time of interview, those with previous RT-PCR testing, pregnant women, less than 18 years of age and those who were unable to give informed consent were excluded.

The study team conducted an ocular inspection of the swabbing sites a day prior to the actual swabbing of the study participants. Collection sites in open spaces and with good air exchange were identified. Three (3) swabbing booths were provided by PCMC. These swabbing booths are made of an acrylic barrier which minimized contact and provided aerosol protection. The swabbing team consisted of three (3) specimen collectors and 3 supervising consultants. The participants' waiting area were designated in front of the swabbing booths and placed at least 2 meters away. They were large enough to ensure adequate social distancing, at 1 meter apart. Tissue and alcohol dispensers were made available to the study participants. Complete PPEs (closed suit with hood, goggles, 3M N95 mask, shoe cover, double gloves) were worn by the specimen collectors, as prescribed by the CDC (Centers for Disease Control). The swabbing team brought with them yellow trash bags for disposal of PPEs and infectious waste.

A general orientation was conducted, providing information on the swabbing procedure and the pooled testing research. After one-on-one interview and counselling, to make sure that all questions have been satisfactorily answered, the participants who agreed to be part of the study signed an informed consent and fill up a Case Investigation form (CIF). CIFs and Informed consent forms were stored in a locked steel cabinet at the administrative office of the PCMC COVID-19 Testing laboratory. The files are accessible only to the principal investigator and co-investigators.

Swabbing was performed according to standard guidelines and procedures. After swabbing, the VTMs were transported back to the COVID-19 Testing Laboratory of PCMC and UPHDMC following biosafety standards and then stored in the reagent refrigerator until testing.

The samples were accessioned according to standard procedures in each laboratory. They were accessioned according to the date, institution/company in successive numbers. The specimens were then pooled in groups of 5 thus, XXXXXXX -P5A, such that,

- "Accession number" represents the individual specimen accession number

- "Pool Accession Number" (ex.: XXXXXXX P 5 A)

- The first alphanumeric characters represent the desired individual accession code
- "P" stands for pool

- " 5 " represents the number of unique individuals in the pool

- "A" represents the sequence in which A is for the first 5 samples pooled, B for the next 5 samples, and so on.

Based on volume used for experiment 1, the specimens were divided into at least thirteen (13) $50 \mathrm{uL}$ aliquots $(3$ each for no pooling, Dorfman 5, and Dorfman 10, and 4 for Dorfman 20+10). A standard accessioning procedure for the aliquots was used. The specimens were then stored in $-80^{\circ} \mathrm{C}$ freezers until ready for processing. Freeze-thaw cycles were minimized.

For the no pooling method, $50 \mathrm{uL}$ aliquots of all the specimens were tested individually using standard laboratory procedures as per manufacturer's specifications using Sansure Novel Coronavirus (2019-nCoV) Nucleic Acid Diagnostic kit. Extraction was done using Natch CS automated extractor and RT-qPCR was performed on MA6000 PCR machine (China).

For purposes of pooled testing, a negative pool is one that shows no target gene amplification. Any target gene amplification (ORF1 and $\mathrm{N}$ genes) regardless of Ct value, degree of amplification or curve properties (sigmoid or non-sigmoid) will be considered positive. Individual samples will undergo the same interpretation as per manufacturer's specifications.

For the 2-stage Dorfman 5, sample aliquots were pooled in groups of 5 , and each resulting pooled aliquot was tested. All specimens in the pools that tested negative were considered negative. All specimens in the pools that tested positive were then tested individually and classified according to the result of this testing.

For the 3-stage Dorfman 10-5, aliquots were first pooled in groups of 10 and tested. All specimens in the pools that tested negative were considered negative. The specimens in the pools that tested positive were then re-pooled into groups of 5 and each sub-pool of 5 was then tested again. All specimens in the sub pools that tested negative were considered negative. All specimens in the pools that tested positive were then tested individually and classified according to the result of this testing.

For the 4-stage Dorfman 20-10-5, aliquots were first pooled in groups of 20 and tested. All specimens in the pools that tested negative were considered negative. The specimens in the pools that tested positive were then repooled into groups of 10 and each sub pool was then tested. All specimens in the sub pools that tested negative were considered negative. The specimens in the pools that tested positive were then re-pooled into groups of 5 and each sub-pool was again tested. All specimens in the sub pools that tested negative were considered negative. All specimens in the sub-pools that tested positive were then tested individually and classified according to the result of this testing.

The individual and pooled samples underwent nucleic acid extraction and SARS-CoV-2 NAAT by RT-qPCR strictly according to the manufacturer's instructions for 
use and followed strict biosafety guidelines and good clinical laboratory practices. Results were recorded and encoded in electronic data collection forms.

Interpretation of pooled sample results (Experiment 2) A pool that shows no gene target amplification whatsoever in any form except for the internal control, is interpreted as negative for all individual samples in that pool. These negative individual samples are reported as negative.

A pool that shows any target gene amplification, regardless of late or low amplification, or unusual or nonsigmoid amplification, is interpreted as a positive pool and deconvolution is done by testing all samples within that pool individually. Results of the individual runs are interpreted according to the manufacturer's instructions.

This procedure was devised based on Experiment 1 that showed loss of sensitivity in pooled samples by increase in Ct values as much as 4.87 in pools of 20 . We wanted to be able to detect possible pools with positive samples that have low viral loads and high Ct values, thus reducing the loss of sensitivity to the minimum possible.

All the residual sample aliquots that were used in the study were disposed of in biological waste bags and autoclaved prior to disposal in the hospital bio-waste facility.

The official results of the RT-qPCR testing were based on the initial individual runs and were released according to standard operating procedures of PCMC and UPHDMC and following the guidelines of the $\mathrm{DOH}$.

Experiment data collected were audited, managed and analyzed by a data management and analysis unit. All valid data were encoded into a password-protected Microsoft Excel file. Access to the data was restricted to key study personnel and were subjected to the approval of the Project Leader. Manual backups were performed, and copies were made in password-protected external hard drives with AES-256 encryption and kept in a lock-andkey cabinet at the administrative office of the PCMC and UPHDMC COVID-19 Testing laboratories.

\section{RESULTS}

\section{Experiment 1}

In Experiment 1, the reference panel consisted of a total of 36 fresh and frozen samples, previously tested positive using A*STAR Fortitude Kit 2.0 COVID-19 Real-Time PCR Test. Fourteen of these were frozen $\left(-80^{\circ} \mathrm{C}\right)$ samples collected in July 2020 while 22 were fresh samples prospectively collected during the duration of phase 1 and were stored at $4^{\circ} \mathrm{C}$ prior to pooling. Among the fresh samples, 18 have been characterized as weak positive (Ct value greater than 30 but less than 38) and 4 were moderate to strong positives. Among the frozen samples, 7 were weak positives and 7 were moderate to strong positives.

Over-all test sensitivity was observed to fall with increasing dilution (simulating dilution by pooling). It decreased to $83 \%(95 \%$ CI $67 \%$ - 94\%) with pool size of 5 , to $72 \%$ (95\% CI: $55 \%-86 \%$ ) with pool size of 10 and to $67 \%(95 \%$ CI: $49 \%-81 \%$ ) with pool size of 20 . Test sensitivity was

\begin{tabular}{|c|c|c|c|c|c|}
\hline \multirow{2}{*}{ Specimen } & \multicolumn{5}{|c|}{ pool size $=5$} \\
\hline & $\mathbf{N}$ & Pos & Sensitivity & $95 \% \mathrm{Cl}$ & \\
\hline Fresh & 22 & 17 & $77 \%$ & $55 \%$ & $92 \%$ \\
\hline Mod to strong positive & 4 & 4 & $100 \%$ & $40 \%$ & $100 \%$ \\
\hline Weak positive & 18 & 13 & $72 \%$ & $47 \%$ & $90 \%$ \\
\hline Frozen & 14 & 13 & $93 \%$ & $66 \%$ & $100 \%$ \\
\hline Mod to strong positive & 7 & 7 & $100 \%$ & $59 \%$ & $100 \%$ \\
\hline Weak positive & 7 & 6 & $86 \%$ & $42 \%$ & $100 \%$ \\
\hline Total & 36 & 30 & $83 \%$ & $67 \%$ & $94 \%$ \\
\hline \multirow{2}{*}{ Specimen } & \multicolumn{5}{|c|}{ pool size $=10$} \\
\hline & $\mathbf{N}$ & Pos & Sensitivity & \multicolumn{2}{|l|}{$95 \% \mathrm{Cl}$} \\
\hline Fresh & 22 & 15 & $68 \%$ & $45 \%$ & $86 \%$ \\
\hline Mod to strong positive & 4 & 4 & $100 \%$ & $40 \%$ & $100 \%$ \\
\hline Weak positive & 18 & 11 & $61 \%$ & $36 \%$ & $83 \%$ \\
\hline Frozen & 14 & 11 & $79 \%$ & $49 \%$ & $95 \%$ \\
\hline Mod to strong positive & 7 & 7 & $100 \%$ & $59 \%$ & $100 \%$ \\
\hline Weak positive & 7 & 4 & $57 \%$ & $18 \%$ & $90 \%$ \\
\hline Total & 36 & 26 & $72 \%$ & $55 \%$ & $86 \%$ \\
\hline \multirow{2}{*}{ Specimen } & \multicolumn{5}{|c|}{ pool size $=\mathbf{2 0}$} \\
\hline & $\mathbf{N}$ & Pos & Sensitivity & $95 \% \mathrm{Cl}$ & \\
\hline Fresh & 22 & 15 & $68 \%$ & $45 \%$ & $86 \%$ \\
\hline Mod to strong positive & 4 & 4 & $100 \%$ & $40 \%$ & $100 \%$ \\
\hline Weak positive & 18 & 11 & $61 \%$ & $36 \%$ & $83 \%$ \\
\hline Frozen & 14 & 9 & $64 \%$ & $35 \%$ & $87 \%$ \\
\hline Mod to strong positive & 7 & 6 & $86 \%$ & $42 \%$ & $100 \%$ \\
\hline Weak positive & 7 & 3 & $43 \%$ & $10 \%$ & $82 \%$ \\
\hline Total & 36 & 24 & $67 \%$ & $49 \%$ & $81 \%$ \\
\hline
\end{tabular}

observed to be maintained at high levels, as high as $100 \%$, in moderate to strong positive samples, even at pool size of 10. This was seen in both fresh and frozen samples. Test sensitivity in weak positive samples decreased with increasing dilutions, from as high as $86 \%$, to as low as $43 \%$. This trend was seen in both the fresh and frozen samples, with the decline more readily seen in frozen samples compared to fresh ones, $86 \%$ - 57\% - 43\% compared to $72 \%-61 \%-61 \%$. Confidence intervals were calculated using the Clopper-Pearson exact method (Table 2).

The Ct-value was observed to increase with increasing dilution. The mean increase in Ct value was 2.56 (95\% CI: $2.24-2.88$ ) with pool size of $5,3.82$ (95\% CI: $3.47-4.16$ ) with pool size of 10 , and 4.87 (95\% CI: 4.35 - 5.39) with pool size of 20. This increase in Ct value was consistently observed in both fresh and frozen samples and in both weak positive and moderate to strong positive samples. Confidence intervals were calculated using the standard normal distribution (Table 3).

Over-all, pool size of 5, which results in a 1:5 dilution at its worst case, was observed to have the least drop in test sensitivity, and the smallest mean change in Ct value, even for samples containing low viral RNA (weak positive samples).

\section{Experiment 2}

In Experiment 2, a total of 440 asymptomatic volunteer employees of a local supermarket chain were recruited.

SARS-CoV-2 RNA was detected in 12 out of the 440 collected samples, giving an estimated prevalence of 
Table 3. Effect of pooling as simulated by dilution on Ct values

\begin{tabular}{|c|c|c|c|c|}
\hline \multirow[b]{2}{*}{ Specimen } & \multirow[b]{2}{*}{ no. } & \multicolumn{3}{|c|}{ Dilution $=1: 5$} \\
\hline & & $\begin{array}{c}\text { mean Ct } \\
\text { change }\end{array}$ & $\begin{array}{l}\text { Lower } \\
95 \% \mathrm{Cl}\end{array}$ & $\begin{array}{l}\text { Upper } \\
95 \% \mathrm{Cl}\end{array}$ \\
\hline Fresh & 22 & 2.74 & 2.28 & 3.20 \\
\hline Mod to strong positive & 4 & 2.05 & 1.68 & 2.42 \\
\hline Weak positive & 18 & 2.89 & 2.36 & 3.43 \\
\hline Frozen & 14 & 2.28 & 1.91 & 2.65 \\
\hline Mod to strong positive & 7 & 2.31 & 2.01 & 2.61 \\
\hline Weak positive & 7 & 2.24 & 1.53 & 2.94 \\
\hline \multirow[t]{2}{*}{ Total } & 36 & 2.56 & 2.24 & 2.88 \\
\hline & & \multicolumn{3}{|c|}{ Dilution $=1: 10$} \\
\hline Specimen & no. & $\begin{array}{c}\text { mean } \mathrm{Ct} \\
\text { change }\end{array}$ & $\begin{array}{l}\text { Lower } \\
95 \% \mathrm{Cl}\end{array}$ & $\begin{array}{l}\text { Upper } \\
95 \% \mathrm{CI}\end{array}$ \\
\hline Fresh & 22 & 4.12 & 3.65 & 4.59 \\
\hline Mod to strong positive & 4 & 3.90 & 2.74 & 5.05 \\
\hline Weak positive & 18 & 4.17 & 3.64 & 4.70 \\
\hline Frozen & 14 & 3.34 & 2.94 & 3.75 \\
\hline Mod to strong positive & 7 & 3.54 & 2.94 & 4.15 \\
\hline Weak positive & 7 & 3.14 & 2.61 & 3.68 \\
\hline \multirow[t]{2}{*}{ Total } & 36 & 3.82 & 3.47 & 4.16 \\
\hline & & \multicolumn{3}{|c|}{ Dilution $=1: 20$} \\
\hline Specimen & no. & $\begin{array}{c}\text { mean } \mathrm{Ct} \\
\text { change }\end{array}$ & $\begin{array}{l}\text { Lower } \\
95 \% \mathrm{Cl}\end{array}$ & $\begin{array}{l}\text { Upper } \\
95 \% \mathrm{Cl}\end{array}$ \\
\hline Fresh & 22 & 4.87 & 4.37 & 5.38 \\
\hline Mod to strong positive & 4 & 5.11 & 3.45 & 6.77 \\
\hline Weak positive & 18 & 4.82 & 4.30 & 5.34 \\
\hline Frozen & 14 & 4.87 & 3.75 & 5.98 \\
\hline Mod to strong positive & 7 & 5.32 & 3.14 & 7.51 \\
\hline Weak positive & 7 & 4.41 & 3.82 & 5.01 \\
\hline Total & 36 & 4.87 & 4.35 & 5.39 \\
\hline
\end{tabular}

\section{Table 4. Ct values of the 12 positive cases}

\begin{tabular}{ccc}
\hline \multirow{2}{*}{ Positive cases } & \multicolumn{2}{c}{ Ct-values } \\
\cline { 2 - 3 } & $\begin{array}{c}\text { FAM } \\
\text { ORF1ab gene }\end{array}$ & $\begin{array}{c}\text { ROX } \\
\text { N gene }\end{array}$ \\
\hline Case 1 & 34.64 & 31.71 \\
Case 2 & - & 34.90 \\
Case 3 & 39.30 & 34.74 \\
Case 4 & 32.33 & 28.19 \\
Case 5 & 38.95 & 34.26 \\
Case 6 & 34.67 & 31.29 \\
Case 7 & 41.19 & 35.58 \\
Case 8 & 35.48 & 31.01 \\
Case 9 & 37.10 & 33.19 \\
Case 10 & 31.63 & 26.72 \\
Case 11 & 31.86 & 27.26 \\
Case 12 & 33.79 & 28.49 \\
\hline
\end{tabular}

$3 \%(95 \%$ CI: $2 \%-5 \%)$, for the sampled population of asymptomatic employees. Official results were released and sent to $\mathrm{DOH}$ which contacted the individuals for case management including quarantine and contact tracing following standard protocols (Table 4).

Test sensitivity was observed to decrease with increasing pool size. In Dorfman 5-1, only 10 of the 12 positive samples were recovered, resulting in a decrease in sensitivity to $83 \%$ (95\% CI: $52 \%$ - 98\%). In Dorfman 10-5-1, only 7 of the 12 positive samples were recovered, for a sensitivity of $58 \%$ (95\% CI: $28 \%-85 \%)$. And the largest decrease in test sensitivity was seen in Dorfman 20-10-5-1, where only 6 of the 12 positive samples were recovered, for a sensitivity of $50 \%$ (95\% CI: $21 \%$ - 79\%). Test specificity was excellent, estimated at 100\% (95\% CI:99\% - 100\%) across the different Dorfman pooling methods (Table 5 ).

Overall accuracy was observed to be consistently high. In Dorfman 5-1, 438 out of 440 samples were correctly classified for an accuracy of 100\% (95\% CI: 98\% - 100\%). Accuracy was the same in Dorfman 10-5-1, where 435 out of 440 samples were correctly classified for an accuracy of $99 \%$ (95\% CI: 97\%-99\%). The overall accuracy was the same with Dorfman 20-10-5-1, where 434 out of 440 samples were correctly classified, for an accuracy of $99 \%$ (95\% CI: $97 \%$ - 99\%) (Table 5).

Test savings were high and ranged from $69 \%$ to $83 \%$ across the different Dorfman pooling methods. Dorfman 20-105-1 resulted in the highest test savings, consuming only 76 tests to generate results for 440 samples, resulting in test savings of $83 \%$ (95\% CI:79\% - 86\%). Dorfman 10-5-1 came second, requiring 93 tests for a test saving of $79 \%$ (95\% CI: $75 \%-83 \%)$. Dorfman 5-1 showed the least test savings of 69\% (95\% CI: $64 \%-73 \%$ ), needing 138 tests for the 440 samples) (Table 6).

Delays in turn-around times were seen. For positive samples, the turnaround time was from 2 to 4 batch runs. Dorfman 5-1 had the fastest turnaround time for positive samples with TAT of 2 batch runs, followed by Dorfman 10-5-1 with 3 batch runs, and Dorfman 20-10-5-1 with 4 batch runs. For negative samples, the average turnaround time was from 1.09 to 1.44 batch runs. Dorfman 20-10-51 had the longest average turnaround time for negative samples with TAT of 1.44 batch runs. This means that on the average, a proportion of the negative samples will require more than one batch run to be released. Dorfman 10-5-1 had an average TAT of 1.21 batch runs for negative samples, while Dorfman 5-1 had the shortest TAT at 1.09 batch runs. This means that on the average, most of the negative samples tested using Dorfman 5-1 would still be released on the same batch run.

\section{DISCUSSION}

Based on results of Experiment 1, which showed reduction of sensitivity to $77 \%$ in pools of $5,54 \%$ in pools of 10 and $46 \%$ in pools of 20 , it was decided to employ measures to mitigate the loss of sensitivity as in the methodology above, where any pool with any form or magnitude of target gene amplification is considered "positive" and its individual samples were tested individually to determine which sample if any, is positive by the manufacturer's specifications.

Notwithstanding this measure, we still missed some positive cases when using aliquots of $50 \mathrm{ul}$ per individual sample for pooling. It is recommended that we use $200 \mathrm{ul}$ as an individual sample contribution to the pool based on a study done by the Korean Society for Laboratory Medicine. ${ }^{30}$

\section{Analysis of the Ct values of positive cases}

The Ct values of both ORF1ab and N gene targets of the 12 positive cases are analyzed to determine concordance with the findings of Experiment 1. Compared to the individual run $\mathrm{Ct}$ values, there was an increase in $\mathrm{Ct}$ values in all 


\begin{tabular}{|c|c|c|c|c|c|c|c|c|c|c|c|c|c|c|c|}
\hline & \multicolumn{15}{|c|}{ Sensitivity } \\
\hline & \multicolumn{5}{|c|}{ Dorfman 5-1 } & \multicolumn{5}{|c|}{ Dorfman 10-5-1 } & \multicolumn{5}{|c|}{ Dorfman 20-10-5-1 } \\
\hline & $\mathbf{x}$ & $\mathbf{n}$ & $\%$ & $95 \% \mathrm{Cl}$ & & $\mathbf{x}$ & $\mathrm{n}$ & $\%$ & $95 \% \mathrm{Cl}$ & & $\mathbf{x}$ & $\mathrm{n}$ & $\%$ & $95 \% \mathrm{Cl}$ & \\
\hline Site A & 6 & 8 & $75 \%$ & $35 \%$ & $97 \%$ & 3 & 8 & $38 \%$ & $9 \%$ & $76 \%$ & 2 & 8 & $25 \%$ & $3 \%$ & $65 \%$ \\
\hline Site B & 4 & 4 & $100 \%$ & $40 \%$ & $100 \%$ & 4 & 4 & $100 \%$ & $40 \%$ & $100 \%$ & 4 & 4 & $100 \%$ & $40 \%$ & $100 \%$ \\
\hline \multirow[t]{4}{*}{ Total } & 10 & 12 & $83 \%$ & $52 \%$ & $98 \%$ & 7 & 12 & $58 \%$ & $28 \%$ & $85 \%$ & 6 & 12 & $50 \%$ & $21 \%$ & $79 \%$ \\
\hline & \multicolumn{15}{|c|}{ Specificity } \\
\hline & \multicolumn{5}{|c|}{ Dorfman 5-1 } & \multicolumn{5}{|c|}{ Dorfman 10-5-1 } & \multicolumn{5}{|c|}{ Dorfman 20-10-5-1 } \\
\hline & $\mathbf{x}$ & $\mathbf{n}$ & $\%$ & $95 \% \mathrm{Cl}$ & & $\mathbf{x}$ & $\mathrm{n}$ & $\%$ & $95 \% \mathrm{Cl}$ & & $x$ & $\mathrm{n}$ & $\%$ & $95 \% \mathrm{Cl}$ & \\
\hline Site A & 212 & 212 & $100 \%$ & $98 \%$ & $100 \%$ & 212 & 212 & $100 \%$ & $98 \%$ & $100 \%$ & 212 & 212 & $100 \%$ & $98 \%$ & $100 \%$ \\
\hline Site B & 216 & 216 & $100 \%$ & $98 \%$ & $100 \%$ & 216 & 216 & $100 \%$ & $98 \%$ & $100 \%$ & 216 & 216 & $100 \%$ & $98 \%$ & $100 \%$ \\
\hline \multirow[t]{4}{*}{ Total } & 428 & 428 & $100 \%$ & $99 \%$ & $100 \%$ & 428 & 428 & $100 \%$ & $99 \%$ & $100 \%$ & 428 & 428 & $100 \%$ & $99 \%$ & $100 \%$ \\
\hline & \multicolumn{15}{|c|}{ Accuracy } \\
\hline & \multicolumn{5}{|c|}{ Dorfman 5-1 } & \multicolumn{5}{|c|}{ Dorfman 10-5-1 } & \multicolumn{5}{|c|}{ Dorfman 20-10-5-1 } \\
\hline & $\mathbf{x}$ & $\mathrm{n}$ & $\%$ & $95 \% \mathrm{Cl}$ & & $\mathbf{x}$ & $\mathrm{n}$ & $\%$ & $95 \% \mathrm{Cl}$ & & $x$ & $\mathrm{n}$ & $\%$ & $95 \% \mathrm{Cl}$ & \\
\hline Site A & 218 & 220 & $99 \%$ & $97 \%$ & $100 \%$ & 215 & 220 & $98 \%$ & $95 \%$ & $99 \%$ & 214 & 220 & $97 \%$ & $94 \%$ & $99 \%$ \\
\hline Site B & 220 & 220 & $100 \%$ & $98 \%$ & $100 \%$ & 220 & 220 & $100 \%$ & $98 \%$ & $100 \%$ & 220 & 220 & $100 \%$ & $98 \%$ & $100 \%$ \\
\hline Total & 438 & 440 & $100 \%$ & $98 \%$ & $100 \%$ & 435 & 440 & $99 \%$ & $97 \%$ & $100 \%$ & 434 & 440 & $99 \%$ & $97 \%$ & $99 \%$ \\
\hline
\end{tabular}

Table 6. Test savings and turnaround time

\begin{tabular}{|c|c|c|c|c|c|c|c|c|c|}
\hline & \multirow{2}{*}{$\begin{array}{c}\text { Prev } \\
\%\end{array}$} & \multicolumn{2}{|c|}{ Number of tests used } & \multicolumn{4}{|c|}{ Test Savings } & \multirow{2}{*}{$\begin{array}{c}\text { TAT (positives) } \\
\text { batch runs* }\end{array}$} & \multirow{2}{*}{$\begin{array}{c}\text { TAT (negatives) } \\
\text { batch runs* }\end{array}$} \\
\hline & & Baseline & Pooling & $\mathrm{n}$ & $\%$ & $95 \% \mathrm{Cl}$ & & & \\
\hline Dorfman 5-1 & 3 & 440 & 138 & 302 & $69 \%$ & $64 \%$ & $73 \%$ & 2 & 1.09 \\
\hline Site A & 4 & 220 & 74 & 146 & $66 \%$ & $60 \%$ & $73 \%$ & 2 & 1.11 \\
\hline Site B & 2 & 220 & 64 & 156 & $71 \%$ & $64 \%$ & $77 \%$ & 2 & 1.07 \\
\hline Dorfman 10-5-1 & 3 & 440 & 93 & 347 & $79 \%$ & $75 \%$ & $83 \%$ & 3 & 1.21 \\
\hline Site A & 4 & 220 & 43 & 177 & $80 \%$ & $75 \%$ & $85 \%$ & 3 & 1.18 \\
\hline Site B & 2 & 220 & 50 & 170 & $77 \%$ & $71 \%$ & $83 \%$ & 3 & 1.24 \\
\hline Dorfman 20-10-5-1 & 3 & 440 & 76 & 364 & $83 \%$ & $79 \%$ & $86 \%$ & 4 & 1.44 \\
\hline Site A & 4 & 220 & 29 & 191 & $87 \%$ & $82 \%$ & $81 \%$ & 4 & 1.29 \\
\hline Site B & 2 & 220 & 47 & 173 & $79 \%$ & $73 \%$ & $84 \%$ & 4 & 1.59 \\
\hline
\end{tabular}

Table 7. Summary of results for Experiment 2

\begin{tabular}{|c|c|c|c|c|c|c|c|c|c|}
\hline & $\begin{array}{c}\text { Prev } \\
\%\end{array}$ & $\begin{array}{c}\text { Tests } \\
\mathbf{n}\end{array}$ & Sensitivity & Specificity & Accuracy & Tests & $\begin{array}{c}\text { Savings } \\
\%\end{array}$ & $\begin{array}{c}\text { TAT (positives) } \\
\text { batch runs* }\end{array}$ & $\begin{array}{c}\text { TAT (negatives) } \\
\text { batch runs* }\end{array}$ \\
\hline Dorfman 5-1 & 3 & 440 & $83 \%$ & $100 \%$ & $100 \%$ & 138 & $69 \%$ & 2 & 1.09 \\
\hline Site A & 4 & 220 & $75 \%$ & $100 \%$ & $99 \%$ & 74 & $66 \%$ & 2 & 1.11 \\
\hline Site B & 2 & 220 & $100 \%$ & $100 \%$ & $100 \%$ & 64 & $71 \%$ & 2 & 1.07 \\
\hline Dorfman 10-5-1 & 3 & 440 & $58 \%$ & $100 \%$ & $99 \%$ & 93 & $79 \%$ & 3 & 1.21 \\
\hline Site $A$ & 4 & 220 & $38 \%$ & $100 \%$ & $98 \%$ & 43 & $80 \%$ & 3 & 1.18 \\
\hline Site B & 2 & 220 & $100 \%$ & $100 \%$ & $100 \%$ & 50 & $77 \%$ & 3 & 1.24 \\
\hline Dorfman 20-10-5-1 & 3 & 440 & $50 \%$ & $100 \%$ & $99 \%$ & 76 & $83 \%$ & 4 & 1.44 \\
\hline Site A & 4 & 220 & $25 \%$ & $100 \%$ & $97 \%$ & 29 & $87 \%$ & 4 & 1.29 \\
\hline Site B & 2 & 220 & $100 \%$ & $100 \%$ & $100 \%$ & 47 & $79 \%$ & 4 & 1.59 \\
\hline
\end{tabular}

pool sizes. Three cases had ORF1ab Ct values close to 40 (37.1, 38.95 and 39.3) which did not allow for a definite value when subtracted from the Ct cut-off of 40 and were excluded from the analysis of ORFlab Ct values. Two cases did not show ORF1ab amplification and were also not included in the calculations. (Table 4).

In the pools of 5 , the $\mathrm{Ct}$ values of ORF1ab and $\mathrm{N}$ gene targets were higher by an average of 0.75 and 2.67 respectively, for an average increase of 2.23. In pools of 10 , the Ct values rose by 4.46 and 3.22 respectively with an average of 3.63 . The corresponding changes were 4.20 and 3.54 in pools of 20 with an average of 3.67 (Table 8 ).
These findings are consistent with the Experiment 1 changes in Ct values as well as in previously cited studies which indicate some loss of sensitivity when samples are pooled, especially with Ct values over 35. However, due to the small sample size of positive cases, determination of loss of sensitivity will be best seen in Experiment 1 .

The yield was best with pools of 5 . Pools of 10 and 20 did not pick up many of the individually positive samples in the original baseline run, especially those with high Ct values. These results are consistent with the observations in Experiment 1, thus, our recommendation is to use pools of 5 in routine testing, based on sensitivity, as 


\begin{tabular}{|c|c|c|c|c|c|c|}
\hline \multirow[b]{2}{*}{ Positive cases } & \multicolumn{2}{|c|}{ Pools of 5} & \multicolumn{2}{|c|}{ Pools of 10} & \multicolumn{2}{|c|}{ Pools of 20} \\
\hline & $\begin{array}{c}\text { FAM } \\
\text { ORF1ab gene }\end{array}$ & $\begin{array}{c}\text { ROX } \\
\text { N gene }\end{array}$ & $\begin{array}{c}\text { FAM } \\
\text { ORF1ab gene }\end{array}$ & $\begin{array}{c}\text { ROX } \\
\text { N gene }\end{array}$ & $\begin{array}{c}\text { FAM } \\
\text { ORF1ab gene }\end{array}$ & $\begin{array}{c}\text { ROX } \\
\text { N gene }\end{array}$ \\
\hline Case 1 & - & 4.39 & 5.02 & 4.02 & - & 5.39 \\
\hline Case 2 & - & 3.10 & - & - & - & 0.99 \\
\hline Case 3 & - & 3.26 & - & - & - & 1.15 \\
\hline Case 4 & - & 8.00 & - & 6.72 & - & 7.70 \\
\hline Case 6 & - & 5.32 & - & 5.16 & - & - \\
\hline Case 7 & - & 1.03 & - & 0.87 & - & - \\
\hline Case 9 & - & 0.19 & - & 4.33 & - & 4.08 \\
\hline Case 10 & 1.46 & 0.77 & 2.02 & 1.98 & 5.73 & 2.78 \\
\hline Case 11 & 0.30 & -0.17 & 7.39 & 0.85 & 2.66 & 1.62 \\
\hline Case 12 & 0.48 & 0.81 & 3.40 & 1.84 & - & 4.58 \\
\hline Average Ct-value change & 0.75 & 2.67 & 4.46 & 3.22 & 4.20 & 3.54 \\
\hline Combined Average & \multicolumn{2}{|c|}{2.23} & \multicolumn{2}{|c|}{3.63} & \multicolumn{2}{|c|}{3.67} \\
\hline
\end{tabular}

well as comparable savings in reagents and better turnaround time.

Recent evidence, however, shows that Ct values 35 and over are associated with low viral loads or even viral remnants in persons who are in the process of recovery in which case they are no longer infectious. ${ }^{31}$ We need to balance our expectations of pooled testing with its expected slight loss of sensitivity with this in mind. This consideration further supports the expanded use of pooled testing in order to curb transmission in the community since we will detect those who have high viral loads ( $\mathrm{Ct}$ values 25 or less) without loss of sensitivity at these levels.

Mina et al., argues thus, "The tests we need are fundamentally different from the clinical tests currently being used, and they must be evaluated differently. Clinical tests are designed for use with symptomatic people, do not need to be low-cost, and require high analytic sensitivity to return a definitive clinical diagnosis given a single opportunity to test. In contrast, tests used in effective surveillance regimens intended to reduce the population prevalence of a respiratory virus need to return results quickly to limit asymptomatic spread and should be sufficiently inexpensive and easy to execute to allow frequent testing - multiple times per week. Transmission of SARS-CoV-2 appears to occur days after exposure, when the viral load peaks. This timing increases the importance of high test frequency, because the test must be used at the beginning of an infection to stop onward spread, and reduces the importance of achieving the very low molecular limits of detection of the standard tests." ${ }^{11}$

While Mina et al., claims that the traditional RT-PCR test fails due to its exquisite sensitivity, the use of pooled testing with its slight loss of sensitivity, test savings and applicability to the asymptomatic population lends it well to its being used for frequent testing in order to catch infectious individual in a timely manner. ${ }^{31}$

To quote Mina et al., once again, "A regimen of regular testing works as a sort of Covid-19 filter, by identifying, isolating, and thus filtering out currently infected persons, including those who are asymptomatic. Measuring the sensitivity of a testing regimen or filter requires us to consider a test in context: how often it's used, to whom it's applied, when in the course of an infection it works, and whether its results are returned in time to prevent spread." ${ }_{11}$

\section{Analysis of Turnaround Times}

Compared to individual runs, a slight delay in turnaround time is seen in pooled testing, which necessitates repeat individual testing of samples in positive pools. This is to be expected and is more pronounced in the 3 stage pooling schemes. However, with proper time management (scheduling of runs within the day to accommodate deconvolution), the delay can be minimized. In return, more subjects can be tested with pooled methods. More positive individuals thus can be identified and isolated and their contacts traced.

Laboratories that will engage in pooled testing should also allot more human resources for pooled testing since some laboratories are running at full capacity and cannot accommodate the additional testing required. More work shifts can be added if not already operating on a 24-hour basis.

The pre-analytical phase can be a source of delays and an increase in turnaround time will ensue if the specimen collection, handling and transport are not properly organized and collection staff are not trained in proper methods for such. We have devised a training module for specimen collection, handling and transport to manage the potential problems that have been identified during the pre-analytical phase of the study.

\section{Analysis of Test Savings}

In all pool sizes studied, test savings were substantial and showed progressive increase with bigger pool sizes. Again, this is expected since the positivity rate is low (3\%). Even at the lowest savings in pools of 5, 69\% savings is seen. However, we must caution that savings is a function of positivity rate and savings will decrease as positivity/ prevalence rates increase. The table below illustrates the potential savings at different prevalence rates. To mitigate the reduction of savings, samples from a cluster or sub-group should be pooled together. For example, subjects from the same household or work force in a particular location should be pooled together rather than mixed with other groups. 


\begin{tabular}{|c|c|c|c|c|c|c|}
\hline \multirow[t]{2}{*}{ Prevalence } & \multicolumn{3}{|c|}{$\begin{array}{c}2 \text { stage Dorfman } \\
\text { Pool size }\end{array}$} & \multicolumn{3}{|c|}{$\begin{array}{c}3 \text { stage Dorfman } \\
\text { Pool sizes }\end{array}$} \\
\hline & 5 & 10 & 20 & $10 \& 5$ & $20 \& 10$ & $25 \& 5$ \\
\hline $1 \%$ & 75 & 80 & 77 & 83 & 84 & 87 \\
\hline $2 \%$ & 71 & 72 & 62 & 77 & 73 & 78 \\
\hline $3 \%$ & 66 & 64 & 49 & 71 & 64 & 71 \\
\hline $4 \%$ & 61 & 56 & 39 & 65 & 56 & 65 \\
\hline $5 \%$ & 57 & 50 & 31 & 59 & 48 & 59 \\
\hline $6 \%$ & 53 & 44 & 24 & 54 & 42 & 54 \\
\hline $7 \%$ & 49 & 38 & 19 & 49 & 36 & 49 \\
\hline $8 \%$ & 46 & 34 & 14 & 44 & 31 & 44 \\
\hline $9 \%$ & 42 & 29 & 10 & 40 & 26 & 40 \\
\hline $10 \%$ & 39 & 25 & 7 & 36 & 21 & 37 \\
\hline $11 \%$ & 36 & 21 & 5 & 32 & 17 & 33 \\
\hline $12 \%$ & 33 & 18 & 3 & 28 & 14 & 29 \\
\hline $13 \%$ & 30 & 15 & 1 & 25 & 10 & 26 \\
\hline $14 \%$ & 27 & 12 & 0 & 21 & 8 & 24 \\
\hline $15 \%$ & 24 & 9 & -1 & 18 & 5 & 21 \\
\hline $16 \%$ & 22 & 7 & -2 & 15 & 3 & 18 \\
\hline $17 \%$ & 20 & 6 & -3 & 13 & 1 & 15 \\
\hline $18 \%$ & 17 & 4 & -3 & 10 & -1 & 13 \\
\hline $19 \%$ & 15 & 2 & -4 & 7 & -3 & 11 \\
\hline $20 \%$ & 13 & 1 & -4 & 5 & -4 & 9 \\
\hline
\end{tabular}

*10,000 batches of 100 specimens each were simulated using the R Programming Language for Statistical Analysis

The amount of savings possible with pooled testing is its main strength. Expanded testing will not be possible without reducing costs of testing and pooled testing actually facilitates more testing. However, to properly manage expectations, preliminary evaluation of the current positivity/prevalence rate of the target populations need to be done. If there is no available data for this, an initial smaller pilot project to determine the positivity rate in the different populations to be tested with the pooled method and the pooled testing adjusted accordingly (Table 9).

\section{Study limitations}

Experiment 1 focused on PCR positive samples with Ct values between 30 to 38 with only a few specimens representing medium and high viral loads. Per the protocol, pooling was only simulated by dilution with transport media and not actual pooling with PCR negative samples to demonstrate loss in sensitivity.

In Experiment 2, only 12 positive cases were captured which, although a good thing as it ensured that the pooling methods will perform optimally, it resulted in wide interval estimates for the test sensitivity. In general, estimation of test sensitivity is best done with at least 50 known positives. The test sensitivity estimated in Experiment 1 may be a more reliable basis for policy making. In addition, the estimate of the overall accuracy in Experiment 2 would be a more reliable metric as can be seen in the narrow $95 \%$ confidence interval widths of the estimates.

Calculation of the test savings were based solely on the number of tests that were consumed during the testing. A more comprehensive test saving calculation would include tests consumed due to repeats due to any reason, and for running quality controls. We are of the opinion, however, that in the long run, the raw number of tests saved will be an important driver for the overall test and cost savings.

The turnaround time evaluation in this study is based on actual testing hours per run, such that samples in the positive pools were included in the next run. This also does not take into full consideration that larger pool sizes will allow for more samples to be tested in any given batch, which will increase the total number of samples tested per day. The delay due to the increase in turnaround time, thus, can be compensated by the total increase in testing capacity provided by pooling samples.

\section{Sources of potential bias and generalizability}

Experiment 1 of this study involved evaluating the effect of pooling on test sensitivity on low amplification samples. Although this may, at first thought, be unrealistic and not representative of actual samples that will be encountered in day to day laboratory operations, using low amplification samples is a good strategy to "stress test" the pooling method and evaluate its performance in extreme situations, where diagnostic errors are more likely to happen and where decision dilemmas frequently occur.

\section{Generalizability}

The results of this study are generalizable to other laboratories in as much as the same protocol will be used on the same target tested population. It is recommended to verify that the performance of RT-PCR kits a laboratory uses are at least as good as the performance characteristics of the kits used in this study. Considering the wide range of copy numbers that the different brands of kits can detect (limit of detection [LOD]), it is recommended to use a larger volume per aliquot $(200 \mathrm{uL}$ or more) to detect samples with low viral loads by providing more template for amplification. It is also recommended that any laboratory intending to implement pooling should carefully evaluate the expected test prevalence of Covid-19 in their tested population. This can be done by reviewing the test positivity rates of the laboratory for the past 2-4 weeks. Any shift in the demographic profile of the tested population that the laboratory services should trigger a review of the test positive prevalence. It is also recommended that laboratories intending to perform pooled testing should conduct initial validation studies with their own RT-qPCR test kits and equipment (Annex A and B). The potential applicability of saliva specimens for pooled RT-PCR testing will further expand the ability to test more individuals with the ease in sample collection it offers. ${ }^{32-42}$

\section{Implications for practice}

This pragmatic proof-of concept operational study which demonstrated that Dorfman 5-1 pooling of naso-pharyngeal and oropharyngeal swab samples is a feasible strategy that will result in at least reasonable test savings, with small effects on overall test accuracy and turnaround time.

\section{Intended use and clinical role of pooling}

Overall, taking the effects of Dorfman pooling on test sensitivity, specificity, accuracy, test savings, and turn- 
around time together, as were observed in this study, it would appear that Dorfman 5-1, with pool size of 5 , is the most reasonable pooling method that can be implemented in the laboratory, as long as the test prevalence of COVID-19 is below $10 \%$ (Table 9).

\section{CONCLUSION}

Pooling is a feasible strategy to further increase testing capacity and decrease cost while keeping accuracy at within acceptable levels. A 2-stage Dorfman 5 pooling strategy appears to be the best method, because it has the highest overall accuracy, while still achieving acceptable test savings, and turnaround time. Pooling of nasopharyngeal and oropharyngeal swab samples prior to RT-qPCR testing may be considered by select molecular diagnostic laboratories to further increase testing capacity and at the same time reduce the cost of testing as a feasible means of adopting more relaxed quarantine schemes.

\section{STATEMENT OF AUTHORSHIP}

All authors certified fulfillment of ICMJE authorship criteria.

\section{AUTHOR DISCLOSURE}

The authors declared no conflict of interest.

\section{FUNDING SOURCE}

This manuscript was sponsored by the Philippine Center for Entrepreneurship Foundation, Inc.

\section{REFERENCES}

1. Fetalco M. DOH confirms 32,000 estimated testing capacity. PTV News; 2020. https://ptvnews.ph/dohconfirms-32000-estimated-testing-capacity/.

2. Department of Health. Philippines now has 100 licensed testing labs. https://www.doh.gov.ph/dohpress\%20release/PHILIPPINES-NOW-HAS-100LICENSED-TESTING-LABS-\%E2\%80\%93-DOH.

3. Ritchie H, Ortiz-Ospina E, Beltekian D, et al. Coronavirus pandemic (COVID-19). Our World in Data; 2020. https://ourworldindata.org/coronavirus.

4. Lancet COVID-19 Commissioners, task Force Chairs and Commission Secretariat. Lancet COVID-19 Commission Statement on the occasion of the 75th session of the UN General Assembly. Lancet. 2020;396(10257):1102-24. PMID: 32941825. PMCID: PMC7489891. https://doi.org/10.1016/S01406736(20)31927-9.

5. Lesmoras M. Pres. Duterte places NCR, Region 2, 3, 4A, Davao City, Pangasinan, Albay under GCQ. PTV News; 2020. https://www.ptvnews.ph/pres-duterteplaces-ncr-region-2-3-4a-davao-city-pangasinanalbay-under-gcq/.

6. Padua Jr. R. Diagnostic testing strategies to manage COVID-19 pandemic: proposed by the Philippine Society of Pathologists, Inc. Philipp J Pathol. 2020; 5(1), 5-8. https://doi.org/10.21141/.PJP.2020.08.

7. Caoili SEC, King RAN, Dungog CC, Reyes JCB, Sarol Jr. JN, de Castro RJ. Sample pooling in a resource- limited situation. PAASE Bulletin 34. On PAASE strategic action group 3: mass testing \& fast-tracking. 2020.

8. Shani-Narkiss H, Gilday OD, Yayon N, Landau ID. Efficient and practical sample pooling for highthroughput PCR diagnosis of COVID-19. medRxiv. 2020. https://doi.org/10.1101/2020.04.06.20052159.

9. Abdalhamid B, Bilder CR, McCutchen EL, et al. Assessment of specimen pooling to conserve SARS CoV2 testing resources. Am J Clin Pathol. 2020;153(6):7158. PMID: 32304208. PMCID: PMC7188150. https:// doi.org/10.1093/ajcp/aqaa064.

10. Bilder CR, Tebbs JM. Pooled-testing procedures for screening high volume clinical specimens in heterogeneous populations. Stat Med. 2012;31(27): 3261-8. PMID: 22415972. PMCID: PMC3500568. https://doi.org/10.1002/sim.5334.

11. Deckert A, Bärnighausen T, Kyei NN. Stimulation of pooled-sample analysis strategies for COVID-19 mass testing. Bull World Health Organ. 2020;98(9): 590-8. PMID: 33012859. PMCID: PMC7463190. https://doi.org/10.2471/BLT.20.257188.

12. Hirotsu Y, Maejima M, Shibusawa M, et al. Pooling RTPCR test of SARS-CoV-2 for large cohort of 'healthy' and infection-suspected patients: a prospective and consecutive study on 1,000 individuals. medRxiv. 2020 https://doi.org/10.1101/2020.05.04.20088146.

13. Hogan CA, Sahoo MK, Pinsky BA. Sample pooling as a strategy to detect community transmission of SARS-CoV-2. JAMA. 2020;323(19):1967-9. PMID: 32250394. PMCID: PMC7136853. https://doi.org/ 10.1001/jama. 2020.5445.

14. Perchetti GA, Sullivan KW, Pepper G, et al. Pooling of SARS-CoV-2 samples to increase molecular testing throughput. J Clin Virol. 2020;131:104570. PMID: 32805524. PMCID: PMC7396208. https://doi.org/ 10.1016/j.jcv.2020.104570.

15. Mulu A, Dawit HA, Fekadu A, et al. Evaluation of Sample Pooling for Screening of SARS-CoV-2. medRxiv. 2020. https://doi.org/10.1101/2020.06.10. 20123398.

16. Litvak E, Tu XM, Pagano M. Screening for the presence of a disease by pooling sera samples. J Am Stat Assoc. 1994;89(426):424-34. https://doi.org/ $10.1080 / 01621459.1994 .10476764$

17. Noriega R, Samore MH. Increasing testing throughput and case detection with a pooled-sample Bayesian approach in the context of COVID-19. bioRxiv. 2020. https://doi.org/10.1101/2020.04.03.024216.

18. Phatarfod RM, Sudbury A. The use of a square array scheme in blood testing. Stat Med. 1994;13(22): 2337-43. PMID: 7855467. https://doi.org/10.1002/ sim.4780132205.

19. Shental N, Levy S, Wuvshet V, et al. Efficient high throughput SARS-CoV-2 testing to detect asymptomatic carriers. medRxiv. 2020. https:/doi. org/10.1101/2020.04.14.20064618.

20. Sterrett A. On the detection of defective members of large populations. Ann Math Stat. 1957;28(4):1033-6. https://www.jstor.org/stable/2237067.

21. Torres I, Albert E, Navarro D. Pooling of nasopharyngeal swab specimens for SARS-CoV-2 detection by RT-PCR. J Med Virol. 2020;92(11): 
2306-7. PMID: 32369202. PMCID: PMC7267454. https://doi.org/10.1002/jmv.25971.

22. Van TT, Miller J, Warshauer DM, et al. Pooling nasopharyngeal/throat swab specimens to increase testing capacity for influenza viruses by PCR. J Clin Microbiol. 2012;50(3):891-6. PMID: 22205820. PMCID: PMC3295167. https://doi.org/10.1128/JCM. 05631-11.

23. Yelin I, Aharony N, Tamar ES, et al. Evaluation of COVID-19 RT-qPCR test in multi-sample pools. Clin Infect Dis. 2020;71(16):2073-8. PMID: 32358960. PMCID: PMC7197588. https://doi.org/10.1093/cid/ ciaa531.

24. Lohse S, Pfuhl T, Berkó-Göttel, et al. Pooling of samples for testing for SARS-CoV-2 in asymptomatic people. Lancet Infect Dis. 2020;20(11):1231-2. PMID: 32530425. PMCID: PMC7194818. https://doi. org/10.1016/S1473-3099(20)30362-5.

25. Dorfman R. The detection of defective members of large populations. Ann Math Stat. 1943;14(4):43640. https://webpages.tuni.fi/uta_statistics/tilasto/liskiarkisto/mtt-perusteet 10/mttp-kurssi 10/Materiaalia/ Dorfman-Ann1943.pdf.

26. Roth WK. History and future of nucleic acid amplification technology blood donor testing. Transfus Med Hemother. 2019;46:67-75. https://doi. org/10.1159/000496749.

27. US Department of Health and Human Services Food and Drug Administration Center for Biologics Evaluation and Research. Guidance for industry: use of nucleic acid tests on pooled and individual samples from donors of whole blood and blood components (including source plasma and source leukocytes) to adequately and appropriately reduce the risk of transmission of HIV-1 and HCV;2004. https://www. fda.gov/media/124349/download.

28. Seli HM, ElBashaar MA,. ElWakil SG. Donor minipool NAT screening for HBV, HCV and HIV: a 2-year experience in a private hospital in Saudi Arabia. Comp Clin Pathol. 2014;23:1125-32. https://doi.org/10.1007/ s00580-013-1751-3.

29. Department of Health. Updated interim guidelines on expanded testing for COVID-19. 2020. https://www. doh.gov.ph/node/22681.

30. Kim SY, Lee J, Sung H, et al. Pooling upper respiratory specimens for rapid mass screening of COVID-19 by real-time RT-PCR. Emerg Infect Dis. 2020;26(10): 2469-72. PMID: 32844739. PMCID: PMC7510748. https://doi.org/10.3201/eid2610.201955.

31. Mina MJ, Parker R, Larremore DB. Rethinking Covid-19 Test sensitivity - a strategy for containment. New Engl J Med. 2020;383(22):e120. PMID: 32997903. https://doi.org/0.1056/NEJMp2025631.

32. Ceron JJ, Lamy E, Martinez-Subiela S, et al. Use of saliva for diagnosis and monitoring the SARS-CoV-2: a general perspective. J Clin Med. 2020;9(5):1491. PMID: 32429101. PMCID: PMC7290439. https://doi. org/10.3390/jcm9051491.

33. Czumbel LM, Kiss S, Farkas N, et al. Saliva as a candidate for COVID-19 diagnostic testing: a metaanalysis. Front Med (Lausanne). 2020;7:465. PMID: 32903849. PMCID: PMC7438940. https://doi.org/ 10.3389/fmed.2020.00465.

34. Fakheran, O, Dehghannejad M, Khademi A. Saliva as a diagnostic specimen for detection of SARS-CoV-2 in suspected patients: a scoping review. Infect Dis Poverty 2020;9(1):100. PMID: 32698862. PMCID: PMC7374661. https://doi.org/10.1186/s40249-02000728-w.

35. Iwasaki S, Fujisawa S, Nakakubu S, et al. Comparison of SARS-CoV-2 detection in nasopharyngeal swab and saliva. J Infect. 2020;81(2):e145-7. PMID: 32504740. PMCID: PMC7270800. https://doi.org/10.1016/j.jinf. 2020.05.071.

36. Ott IM, Strine MS, Watkins AE, et al. Simply saliva: stability of SARS-CoV-2 detection negates the need for expensive collection devices. medRxiv 2020.08.03.20165233. PMID: 32793924. PMCID: PMC7418742. https://doi.org/10.1101/2020.08.03.201 65233.

37. Pasomsub E, Watcharananan SP, Boonyawat K, et al. Saliva sample as a non-invasive specimen for the diagnosis of coronavirus disease-2019 (COVID-19): a cross-sectional study. Clin Microbiol Infect 2020. S1198-743X(20)30278-0. PMID: 32422408. PMCID: PMC7227531. https://doi.org/10.1016/j.cmi. 2020.05.001.

38. Ranoa DRE, Holland RL, Alnaji FG, et al. Salivabased molecular testing for SARS-CoV-2 that bypasses RNA extraction. bioRxiv. 2020. https://doi. org/10.1101/2020.06.18.159434.

39. Vogels CBF, Brackney D, Wang J, et al. SalivaDirect: simple and sensitive molecular diagnostic test for SARS-CoV2 surveillance. medRxiv. 2020. https://doi. org/10.1101/2020.08.03.20167791.

40. UI receives FDA approval for saliva-based COVID-19 test first offered on campus. 2020. https://www.wcia. $\mathrm{com} /$ news/ui-receives-fda-approval-for-saliva-basedtest-covid-19-test-first-offered-on-campus/.

41. Wyllie AL, Fournier J, Casanovas-Massana A, et al. Saliva or nasopharyngeal swab specimens for detection of SARS-CoV-2. N Engl J Med. 2020;383(13): 1283-6. PMID: 32857487. PMCID: PMC7484747. https://doi.org/10.1056/NEJMc2016359.

42. Xu J, Li Y, Gan F, Du Y, Yao Y. Salivary glands: potential reservoirs for COVID-19 asymptomatic Infection. J Dent Res. 2020; 99(8):989. PMID: 32271653. https://doi.org/10.1177/0022034520918518.

\footnotetext{
Disclaimer: This journal is OPEN ACCESS, providing immediate access to its content on the principle that making research freely available to the public supports a greater global exchange of knowledge. As a requirement for submission to the PJP, all authors have accomplished an AUTHOR FORM, which declares that the ICMJE criteria for authorship have been met by each author listed, that the article represents original material, has not been published, accepted for publication in other journals, or concurrently submitted to other journals, and that all funding and conflicts of interest have been declared. Consent forms have been secured for the publication of information about patients or cases; otherwise, authors have declared that all means have been exhausted for securing consent.
} 


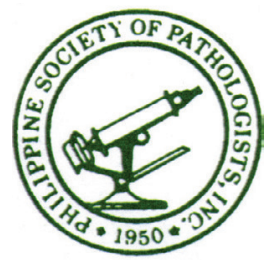

\author{
PHILIPPINE SOCIETY OF PATHOLOGISTS, INC. \\ A Specialty Division of the Philippine Medical Association \\ 114 Malakas Street, Diliman, Quezon City \\ Tel No. 738-68-14; 697-4923 TelFax No 920-31-92 \\ E-mail pspinc1950@yahoo.com
}

\title{
ANNEXA. POOLED PCR TESTING WORK INSTRUCTIONS AND GENERAL GUIDELINES
}

\section{INTRODUCTION}

It has been proven that sample pooling can reduce sensitivity of RT-PCR assays for SARS-CoV-2 by several magnitudes i.e., Ct values may increase from 2.56 up to 4.87 higher. This may lead to false negatives and impact virus containment measures. Thus, it is important to make revisions to the interpretation of pooled samples to negate the impact of the loss of sensitivity.

The approach to specimen pooling shall be through pooling of standard volume aliquots of transport media with each containing a single patient sample.

Specimens obtained from different sources or different sample types should not be pooled together, i.e., only nasopharyngeal samples shall be pooled with nasopharyngeal samples, and so on.

The volume of the samples initially collected from an individual must be sufficient for both the pooled testing and individual follow up testing, if needed. This will prevent the need for a second sample collection.

\section{SPECIMEN RECEPTION}

1.1. The laboratory receptionist shall:

1.1.1. Receive the specimen in the receiving room/reception area and check the appropriateness of the transport conditions and the packaging of the specimens.

1.1.1.1. Packaging specimens into groups of five (5) samples must be strictly adhered.

1.1.1.2. If specimens are not received in groups of five (5) samples, these events must be documented and reported to collection teams and/or the source of specimens

1.1.2. Cross-checks the details of the patient on the line list/master list, laboratory request form, Case Investigation Form (CIF) and PhilHealth Form CF2 (whichever applies), making sure that the patient name and a second identifier matches the accompanying document.

1.1.3. Encode in an electronic line list which will then be transmitted to the staff in charge of specimen handling and inactivation.

1.1.4. Retain and file the original copies in the receiving room and store them appropriately based on existing protocols.

1.2. The medical technologist/analyst shall:

1.2.1. Receive the triple-packaged samples from the reception area/laboratory receptionist together with the master list/line list, thru a pass box and place it inside a biological safety cabinet.

1.2.2. Disinfect the outer container/box with $70 \%$ ethanol and wipe with tissue paper

1.2.3. Disinfect the inner/second container with $70 \%$ ethanol and wipe with tissue paper

1.2.4. Remove the samples from the transport box and Individually inspect the samples

1.2.5. Individually inspect the samples and asses the specimen integrity via a set criterion for acceptance and rejection, together with a second analyst/laboratory aide

1.2.6. Verify the completeness of data in the individual labels on the specimens based on the submitted line list/master list

1.2.7. Asses the specimen integrity via a set criterion for acceptance and rejection, together with a second analyst/laboratory aide, taking note of the following acceptance criteria:

1.2.7.1. Swab/s are present in the collection tube

1.2.7.2. Test requisition with patient name and a second identifier

1.2.7.3. Tube label with patient name and a second identifier

1.2.7.4. Collection tube has no leaks and that the cap is intact

1.2.7.5. The specimen is within stability criteria

1.2.8. Records any rejected specimens and submits this document to the receptionist and laboratory manager. Rejected specimens shall then be excluded from the pool batch. 


\section{SPECIMEN ACCESSIONING}

2.1. Together with a second analyst in the specimen preparation room, the analyst shall:

2.1.1. Gather specimens into groups of five (5) unique individuals per pool by convenience sampling, or as they are received from the reception area.

2.1.2. Assign a set of unique pooling accession numbers (See attached template) alongside the unique individual accession numbers. An example would be:

\begin{tabular}{|c|c|}
\hline Accession Number & Pool Accession Number \\
\hline A0801PE001 & \\
\hline A0801PE002 & \\
\hline A0801PE003 & \\
\hline A0801PE004 & \\
\hline A0801PE005 & \\
\hline
\end{tabular}

"Accession number" represents the individual unique specimen accession number

"Pool Accession Number" (ex.: XXXXXXX P 5 A)

- The first alphanumeric characters represent the desired accession code

- "P" stands for pool

- " 5 " represents the number of unique individuals in the pool

- "A" represents the sequence in which $A$ is for the first 5 samples pooled, $B$ for the next 5 samples, and so on.

The medical technologist who performed the accessioning relays to another medical technologist in the reagent preparation room the total number of specimens for running, taking into account the number of controls, and that each pool of 5 samples are accounted for a single run, and thus the reagents needed should correspond to only one test. He/she then waits for the cue from the medical technologist in the specimen preparation room when to start the reagent preparation, making sure there are no delays and that only freshly prepared reagents are used.

\section{POOLING PROCEDURE}

3.1. The recommended dilution factor should be carefully applied considering the characteristics of the target population, and this protocol recommends pools of 5 .

3.2. The medical technologist analyst shall:

3.2.1. Identify the samples together with another analyst (buddy)

3.2.2. Arrange the specimens into 5 samples per row and a cryovial labeled with the corresponding pool (i.e., P5A, P5B and so on)

3.2.3. Fill out a printed PCR map template with the accession number corresponding to each pool

3.2.4. With a calibrated pipette with filtered pipette tip, transfer $200 \mathrm{uL}$ from each of the 5 individual samples into a $2.0 \mathrm{~mL}$ cryovial tube, making sure that the sample is properly mixed. When aliquoting and mixing with a pipette, collect the same amount from individual samples and mix in a new container. All pipette tips shall be used only once per sample or at each step. If the sample volume to be collected is $200 \mathrm{uL}$ or more, the final volume shall all always be $10 \%$ more than the sample for nucleic acid extraction to make a mixed sample.

3.2.5. Transfer an aliquot from the pool using the volume recommended for existing laboratory protocols for extraction.

3.2.6. Pass or communicate the PCR map to the PCR room.

\section{POOL EXTRACTION AND PCR}

4.1. The medical technologist shall:

4.1.1. Perform sample inactivation according to existing laboratory protocols.

4.1.2. Follow the recommendations of the manufacturer of the nucleic acid extraction reagent, equipment, and PCR reagent for the mixed sample. If the equipment used has a high extraction efficiency, and a large sample volume is used, the possibility of nucleic acid detection in mixed samples is high.

4.1.3. Check the information of the amount of sample used before extraction and the amount and concentration of nucleic acid eluted after extraction.

4.1.4. Store the specimen samples according to existing protocols.

4.1.5. Perform PCR amplification according to existing laboratory protocols.

4.1.6. Set aside and store the pools that test positive, to be individually tested in the next immediate run. 


\section{QUALITY CONTROL IN DIAGNOSTIC PCR LABORATORIES}

5.1. The following are the general guidelines for Quality Control in Diagnostic PCR laboratories for infectious diseases:

5.1.1. Maintain separate areas and dedicated equipment (eg. pipettes, microcentrifuges) and supplies (eg. microcentrifuge tubes, pipette tips, gowns and gloves) for assay reagent setup and handling of extracted nucleic acids.

5.1.2. Workflow must always be from the clean area to the dirty area.

5.1.3. Wear clean disposable gowns and new, previously unworn, powder-free gloves during assay reagent setup and handling of extracted nucleic acids. Change gloves whenever contamination is suspected.

5.1.4. Store primer/probes and enzyme master mix at appropriate temperatures (see package inserts). Do not use reagents beyond their expiry dates.

5.1.5. Keep reagent tubes and reactions capped as much as possible.

5.1.6. Clean and decontaminate surfaces.

5.1.7. Do not bring extracted nucleic acid or PCR products into the assay setup area.

5.1.8. Use aerosol barrier (filtered) pipette tips only.

5.1.9. Use PCR plate strip caps only. Do not use PCR plate sealing film.

5.1.10. Assay controls should be run concurrently with all test samples. If using a commercial kit, check if the following are already included in the kit:

5.1.10.1. PTC - positive template control with an expected Ct value range

5.1.10.2. NTC - negative template control added during RT-qPCR reaction set-up

5.1.10.3. RP - all clinical samples should be tested for human RNAse P (RNP) gene to assess specimen quality

5.1.11. Keep running logs of PTC performance. After each RT-qPCR run of clinical samples, the control Ct values should be recorded.

\section{RESULTS ANALYSIS}

To ensure the absence of non-specific PCR inhibition of a sample, an internal positive amplification control or internal control is included in each specimen. A sample can be interpreted as negative only if the analysis of the internal positive control indicates that the amplification occurred in the reaction tube but no signal from the target reporter dye has been detected.

6.1. The pathologist shall:

6.1.1. Follow the usual validation of negative and positive control samples.

6.1.2. Interpret results according to the following:

6.1.2.1. If the pool tests "negative", report individual samples of that pool as negative or "not detected".

6.1.2.2. Interpret pools as "positive" if at least one gene target shows any form of amplification (late and low amplification, unusual or non-sigmoid curve).

6.1.3. Retest each constituent specimens individually from the pooled samples tagged as "positive" and refer to the individual accession templates described above.

6.1.4. Interpret individual runs according to kit manufacturer's specifications.

6.1.5. Report results of individually-ran samples according to existing laboratory protocols.

\begin{tabular}{|c|c|l|}
\hline \multicolumn{3}{|c|}{ Reporting of Pooled SARS-CoV-2 Testing Results } \\
\hline Result & Interpretations & \multicolumn{1}{c|}{ Actions } \\
\hline Not Detected & Negative & Report each individual in pool as negative \\
\hline Detected & Positive & $\begin{array}{l}\text { Do not report pooled result. Perform diagnostic testing of } \\
\text { individual specimens and report each as "positive" or "negative" }\end{array}$ \\
\hline
\end{tabular}

\section{DECONVOLUTION AND INDIVIDUAL EXTRACTION AND PCR}

When pooled samples test positive, samples in these pools should be identified and tested individually.

7.1. The medical technologist shall:

7.2. Obtain another sample from the original specimen and re-test them individually according to the laboratory and manufacturer's protocol.

7.3. Release individual results and indicate in the report that individual sample testing was done.

\section{RESULTS RELEASE}

8.1. The staff of the COVID-19 testing laboratory shall release the test results as per the laboratory's existing protocols on releasing of RT-PCR results.

8.2. Due to the reduction in analytical sensitivity, a pooling strategy should apply risk mitigation procedures such as indicating in the test result/report that the testing procedure involved specimen pooling. 


\title{
ANNEX B. STANDARD METHOD FOR VERIFICATION OF POOLED TESTING INTERIM GUIDANCE FOR LABORATORIES IN THE COVID-19 LABORATORY NETWORK
}

\author{
As of 26 October 2020
}

\section{INTRODUCTION}

Pooled testing shall be applicable to the performance of SARS-CoV-2 molecular diagnostic tests for the in vitro qualitative detection of RNA from SARS-CoV-2 in respiratory samples for specific target populations only and in line with the Department of Health's latest issuance on testing. Symptomatic patients, as well as people who have high risk exposure to SARS-CoV-2 confirmed cases, shall not be covered by pooled testing.

Pooled testing shall be undertaken only by $\mathrm{DOH}$-selected licensed laboratories of the COVID-19 Laboratory Network only upon review and approval of pooling procedure and method verification data. Only FDA-authorized PCR kits shall be utilized for pooled testing. Pooled testing shall only be performed by qualified proficient laboratory technical staff. The strategy shall only be applied to appropriate target populations with expected low prevalence and low risk. It must be noted that the method may change as more information becomes available.

\section{RATIONALE}

In keeping with international standards of good laboratory practice, any new method introduced by the laboratory shall be subject to verification prior to regular performance.

\section{SCOPE}

The following contains guidance for the standard method for verification of pooled testing as a testing strategy for SARS-CoV-2 PCR as applicable to respiratory specimens.

\section{Objective}

1. To determine the performance, usefulness, practicality, and applicability of pooled testing in a laboratory's particular set-up using its specific PCR reagents, supplies, and laboratory equipment.

2. To verify the method of pooled testing through determination of the percentage agreement between 5-pooled samples and individual samples tested through rRT-PCR prior to adoption of the procedure in the laboratory.

\section{METHOD}

1. Preparation of verification panels

a. Positive pools

i. Using either archived/stored or prospectively collected samples, prepare 20 "positive" pools of five consisting of 80 unique PCR negative samples and 20 PCR positive samples, for a total of 100 samples.

ii. The 20 PCR positive samples shall consist of:

1. $25 \%(n=5)$ within $2-3$ Ct values of the cut-off for the laboratory's PCR assay, to represent low or weak positives

2. $75 \%(n=15)$ with various $\mathrm{Ct}$ values representing high and medium positive samples

iii. Each "positive" 5 -sample pool shall consist of $1 \mathrm{PCR}$ positive sample +4 randomly selected PCR negative samples

b. Negative pools

i. Using either archived/stored or prospectively collected samples, prepare 20 "negative" pools of five consisting of 100 unique PCR negative samples.

ii. If there is sufficient volume, the same negatives used in the preparation of the "positive" pools may be used.

Note: All samples included in the positive and negative pools should have been tested individually using the laboratory's PCR assay, following manufacturer's instructions, with recording of Ct values.

\section{PCR Testing of positive and negative pools}

a. The 20 positive and 20 negative pools shall be tested using the laboratory's PCR assay, following the manufacturer's instructions, with recording of Ct values for each gene target.

b. Ensure that the technical staff conducting the tests on the positive and negative pools are blinded to the results of the individual samples included in each pool. 


\section{Analysis of data}

\section{a. Data tables}

i. Data shall be summarized following the table below, showing the Ct values, interpretation of individual results with corresponding pooled test results.

\begin{tabular}{|c|c|c|c|c|c|}
\hline \multicolumn{3}{|c|}{ Individual Samples } & \multicolumn{3}{|c|}{ Pooled Test Result } \\
\hline Test Result & $\begin{array}{l}\text { Ct Value per } \\
\text { gene target }\end{array}$ & $\begin{array}{c}\text { Result } \\
\text { Interpretation }\end{array}$ & Pool Number & $\begin{array}{l}\text { Ct Value per } \\
\text { gene target }\end{array}$ & $\begin{array}{c}\text { Result } \\
\text { Interpretation }\end{array}$ \\
\hline Sample Lab ID-001 & & & \multirow{5}{*}{ Pool Number-001 } & & \\
\hline Sample Lab ID-002 & & & & & \\
\hline Sample Lab ID-003 & & & & & \\
\hline Sample Lab ID-004 & & & & & \\
\hline Sample Lab ID-005 & & & & & \\
\hline$\ldots$ & & & $\ldots$. & & \\
\hline Sample Lab ID-100 & & & Pool Number- 020 & & \\
\hline
\end{tabular}

b. Percent Agreement (Aggregate)

i. Calculate the percent agreement of the pooled samples with respect to the expected results (i.e., if a positive patient sample was included in the 5-sample pools, the expected result was positive).

\begin{tabular}{|c|c|c|}
\hline Samples Tested Individually & \multicolumn{2}{|c|}{ Pooled Test Final Result (5-sample Pool) } \\
\hline Test Result & Positive & Negative \\
\hline Positive & & \\
\hline Negative & & \\
\hline \multicolumn{2}{|l}{ Positive Percent Agreement $=$}
\end{tabular}

Formula:

No. of positive results in agreement (Pooled test result with individual result) Total number of results $(20)$

c. Percent Agreement (Disaggregated to Ct value range)

i. Using the table below:

\begin{tabular}{|c|c|c|}
\hline $\begin{array}{l}\text { Samples Tested in a } \\
\text { 5-sample Pool }\end{array}$ & \multicolumn{2}{|c|}{$\begin{array}{l}\text { Individual Samples with Ct within } 3 \text { values } \\
\text { within PCR kit cutoff (weak positives) }\end{array}$} \\
\hline Pooled Test Result & Positive & Negative \\
\hline \multicolumn{3}{|l|}{ Positive } \\
\hline \multicolumn{3}{|l|}{ Negative } \\
\hline & \multicolumn{2}{|c|}{ Individual Samples with Ct values $>30$ to $37^{*}$} \\
\hline \multicolumn{3}{|c|}{ (1) } \\
\hline \multicolumn{3}{|l|}{ Negative } \\
\hline & \multicolumn{2}{|c|}{ Individual Samples with Ct values $>20$ to 30} \\
\hline \multicolumn{3}{|l|}{ Positive } \\
\hline \multicolumn{3}{|l|}{ Negative } \\
\hline & \multicolumn{2}{|c|}{ Individual Samples with $\mathrm{Ct}$ values $<20$} \\
\hline \multicolumn{3}{|l|}{ Positive } \\
\hline Negative & & \\
\hline
\end{tabular}

\section{Record Keeping}

The laboratory shall maintain information on the performance of the pooled testing procedure and all method verification data. These records shall be made available for review and inspection upon request.

\section{Submission of Verification Documents}

The accomplished method verification report shall be submitted to the Research Institute for Tropical Medicine for review. 


\section{REFERENCES}

1. US FDA In Vitro Diagnostics Emergency Use Authorizations (EUAs). https://www.fda.gov/medical-devices/ coronavirus-disease-2019-covid-19-emergency-use-authorizations-medical-devices/vitro-diagnosticseuas.

2. Abdalhamid, B., Bilder, C. R., McCutchen, E. L., Hinrichs, S. H., Koepsell, S. A., \& Iwen, P. C. (2020). Assessment of Specimen Pooling to Conserve SARS CoV-2 Testing Resources. American Journal of Clinical Pathology, 153(6), 715-718. https://doi.org/10.1093/ajcp/aqaa0644

3. Bilder, C. R., \& Tebbs, J. M. (2012). Pooled-testing procedures for screening high volume clinical specimens in heterogeneous populations. Statistics in Medicine, 31(27), 3261-3268. https://doi.org/10.1002/sim.5334

4. Caoili, S. E., Cecile Dungog, Jesus Sarol, King, R. A., Reyes, J. C., \& Romulo, de C. (2020). A recommendation for COVID-19 TESTING in LOW- RESOURCE COUNTRIES.

5. Dorfman, R. (1943). The Detection of Defective Members of Large Populations. Annals of Mathematical Statistics, 14(4), 436-440. https://doi.org/10.1214/aoms/1177731363

6. Hirotsu, Y., Maejima, M., Shibusawa, M., Nagakubo, Y., Hosaka, K., Amemiya, K., Sueki, H., Hayakawa, M., Mochizuki, H., \& Omata, M. (2020). Pooling RT-PCR test of SARS-CoV-2 for large cohort of "healthy" and infection-suspected patients: A prospective and consecutive study on 1,000 individuals [Preprint]. Epidemiology. https://doi.org/10.1101/2020.05.04.20088146

7. Hogan, C. A., Sahoo, M. K., \& Pinsky, B. A. (2020). Sample Pooling as a Strategy to Detect Community Transmission of SARS-CoV-2. JAMA, 323(19), 1967. https://doi.org/10.1001/jama.2020.5445

8. Lohse, S., Pfuhl, T., Berkó-Göttel, B., Rissland, J., Geißler, T., Gärtner, B., Becker, S. L., Schneitler, S., \& Smola, S. (2020). Pooling of samples for testing for SARS-CoV-2 in asymptomatic people. The Lancet Infectious Diseases, S1473309920303625. https://doi.org/10.1016/S1473-3099(20)30362-5

9. Noriega, R., \& Samore, M. H. (2020). Increasing testing throughput and case detection with a pooled-sample Bayesian approach in the context of COVID-19 [Preprint]. Microbiology. https://doi. org/10.1101/2020.04.03.024216

10. Shani-Narkiss, H., Gilday, O. D., Yayon, N., \& Landau, I. D. (2020). Efficient and Practical Sample Pooling for High-Throughput PCR Diagnosis of COVID-19 [Preprint]. Public and Global Health. https://doi. org/10.1101/2020.04.06.20052159

11. Shental, N., Levy, S., Skorniakov, S., Wuvshet, V., Shemer-Avni, Y., Porgador, A., \& Hertz, T. (2020). Efficient high throughput SARS-CoV-2 testing to detect asymptomatic carriers [Preprint]. Infectious Diseases (except HIV/AIDS). https://doi.org/10.1101/2020.04.14.20064618

12. Torres, I., Albert, E., \& Navarro, D. (2020). Pooling of Nasopharyngeal Swab Specimens for SARS-CoV-2 detection by RT-PCR [Preprint]. Infectious Diseases (except HIV/AIDS). https://doi. org/10.1101/2020.04.22.20075598

13. Van, T. T., Miller, J., Warshauer, D. M., Reisdorf, E., Jernigan, D., Humes, R., \& Shult, P. A. (2012). Pooling Nasopharyngeal/Throat Swab Specimens To Increase Testing Capacity for Influenza Viruses by PCR. Journal of Clinical Microbiology, 50(3), 891-896. https://doi.org/10.1128/JCM.05631-11

14. Yelin, I., Aharony, N., Shaer Tamar, E., Argoetti, A., Messer, E., Berenbaum, D., Shafran, E., Kuzli, A., Gandali, N., Shkedi, O., Hashimshony, T., Mandel-Gutfreund, Y., Halberthal, M., Geffen, Y., SzwarcwortCohen, M., \& Kishony, R. (2020). Evaluation of COVID-19 RT-qPCR test in multi-sample pools. Clinical Infectious Diseases, ciaa531. https://doi.org/10.1093/cid/ciaa531 


\section{METHOD VERIFICATION REPORT FOR POOLED TESTING (TEMPLATE)}

\begin{tabular}{|c|c|c|c|}
\hline \multicolumn{4}{|l|}{ Date of verification: } \\
\hline \multicolumn{4}{|l|}{ Name of Laboratory: } \\
\hline \multicolumn{4}{|l|}{ Complete Address: } \\
\hline \multicolumn{4}{|l|}{ Laboratory head (Pathologist): } \\
\hline \multicolumn{4}{|l|}{ Chief medical technologist: } \\
\hline Contact information: & E-mail address: & Landline No. & Mobile No. \\
\hline \multicolumn{4}{|c|}{ Part 1: PCR Assay Intended to be used for Pooled Testing Verification } \\
\hline Nucleic Acid Extraction kit & \multicolumn{3}{|c|}{$\begin{array}{l}\text { - Brand/manufacturer: } \\
\text { - Kit description: } \\
\text { - Attach product brochure and Manufacturer's Instructions for Use } \\
\text { - Attach Laboratory's SOP }\end{array}$} \\
\hline Automated Extraction machine & \multicolumn{3}{|c|}{$\begin{array}{l}\text { - Brand/manufacturer: } \\
\text { - Attach product brochure } \\
\text { - Attach Laboratory's SOP for operating the machine }\end{array}$} \\
\hline PCR detection kit & \multicolumn{3}{|c|}{$\begin{array}{l}\text { - Brand/manufacturer: } \\
\text { - Kit description: } \\
\text { - Gene targets: } \\
\text { - Attarmance data available (FIND, WHO, National Regulatory Agency, RITM) } \\
\text { - Attach Laboratory's SOP }\end{array}$} \\
\hline PCR machine & \multicolumn{3}{|c|}{$\begin{array}{l}\text { - Brand/manufacturer } \\
\text { - Attach product brochure } \\
\text { - Attach Laboratory's SOP for operating the machine }\end{array}$} \\
\hline \multicolumn{4}{|c|}{ Part 2: Verification Panel Composition } \\
\hline \multicolumn{4}{|c|}{$\begin{array}{l}\text { Positive pools } \\
\text { Number/quantity? } \\
\text { - Archived/stored or prospectively collected? } \\
\text { - Ct valued/stored, dates of collection, storage condition }\left(2-8^{\circ} \mathrm{C},-20^{\circ} \mathrm{C},-40^{\circ} \mathrm{C},-80^{\circ} \mathrm{C}\right)\end{array}$} \\
\hline \multicolumn{4}{|c|}{$\begin{array}{l}\text { Negative pools } \\
\text { Number/quantity? } \\
\text { Archived/stored or prospectively collected? } \\
\text { - If archived/stored, dates of collection, storage condition }\left(2-8^{\circ} \mathrm{C},-20^{\circ} \mathrm{C},-40^{\circ} \mathrm{C},-80^{\circ} \mathrm{C}\right)\end{array}$} \\
\hline
\end{tabular}

Part 3: Pooling Procedure (Detailed specific steps for pooling undertaken) 


Part 4. Results
Summary table (per gene target)
\begin{tabular}{|c|c|c|c|c|c|}
\hline \multicolumn{3}{|c|}{ Individual Samples } & \multicolumn{3}{c|}{ Pooled Test Result } \\
\hline Test Result & $\begin{array}{c}\text { Ct Value per } \\
\text { gene target }\end{array}$ & $\begin{array}{c}\text { Result } \\
\text { Interpretation }\end{array}$ & \multirow{2}{*}{ Pool Number } & $\begin{array}{c}\text { Ct Value per } \\
\text { gene target }\end{array}$ & $\begin{array}{c}\text { Result } \\
\text { Interpretation }\end{array}$ \\
\hline Sample Lab ID-001 & & \multirow{3}{*}{ Pool Number-001 } & & \\
\hline Sample Lab ID-002 & & & & & \\
\hline Sample Lab ID-003 & & & & & \\
\hline Sample Lab ID-004 & & & & & \\
\hline Sample Lab ID-005 & & & & & \\
\hline & & & & & \\
\hline
\end{tabular}

Percentage Agreement (Aggregate)

\begin{tabular}{|c|l|c|}
\hline Samples Tested Individually & Pooled Test Final Result (5-sample Pool) \\
\hline Test Result & Positive & Negative \\
\hline Positive & & \\
\hline Negative & & \\
\hline
\end{tabular}

Notes:

Percentage Agreement (Disaggregated to $\mathrm{Ct}$ value range)

\begin{tabular}{|c|c|c|}
\hline Samples Tested in a 5-sample Pool & \multicolumn{2}{|c|}{$\begin{array}{l}\text { Individual Samples with Ct within } 3 \text { values } \\
\text { within PCR kit cutoff (weak positives) }\end{array}$} \\
\hline Pooled Test Result & Positive & Negative \\
\hline \multicolumn{3}{|l|}{ Positive } \\
\hline \multicolumn{3}{|l|}{ Negative } \\
\hline & \multicolumn{2}{|c|}{ Individual Samples with Ct values $>\mathbf{3 0}$ to 37} \\
\hline \multicolumn{3}{|c|}{ ( } \\
\hline \multicolumn{3}{|l|}{ Negative } \\
\hline & \multicolumn{2}{|c|}{ Individual Samples with $\mathrm{Ct}$ values $>\mathbf{2 0}$ to $\mathbf{3 0}$} \\
\hline \multicolumn{3}{|l|}{ Positive } \\
\hline \multicolumn{3}{|l|}{ Negative } \\
\hline & \multicolumn{2}{|c|}{ Individual Samples with $\mathrm{Ct}$ values $<20$} \\
\hline \multicolumn{3}{|l|}{ Positive } \\
\hline Negative & & \\
\hline
\end{tabular}

Notes:

Report prepared by:

Technical Staff

Signature over printed name
Approved by:

Head of Laboratory

Signature over printed name 


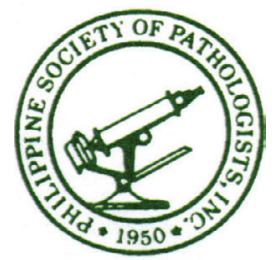

\title{
PHILIPPINE SOCIETY OF PATHOLOGISTS, INC.
}

\author{
A Specialty Division of the Philippine Medical Association \\ 114 Malakas Street, Diliman, Quezon City \\ Tel No. 738-68-14; 697-4923 TelFax No 920-31-92 \\ E-mail pspinc1950@yahoo.com
}

\section{ANNEX C. PHILIPPINE SOCIETY OF PATHOLOGISTS RECOMMENDATION FOR POOLED TESTING}

The Philippine Society of Pathologists Inc. is an organization of physicians specializing in Pathology and Laboratory Medicine with a membership of over 1,000. The society and its members, with specialization and sub-specialization in various areas of the diagnostic field, including Molecular Pathology and Immunopathology, stands in solidarity with the rest of the nation in its fight against SARS-CoV-2.

The statements issued are based on the preliminary findings of the research study "An Evaluation of Pooling Strategies for RT-qPCR testing for SARS-CoV-2 Infection: A pragmatic parallel multi-site operational study by the PSP Inc." This was conducted in Research Institute of Tropical Medicine, Philippine Children's Medical Center, and University of Perpetual Help DALTA Medical Center as principal investigator sites. The research was supported and funded by Philippine Center for Entrepreneurship Inc.

Based on our preliminary research findings, sample pooling can be used as a strategy to enhance COVID-19 testing to increase the number of tests conducted in the country. It will conserve much needed resources, improve turnaround time, and make the test affordable. More importantly as a surveillance testing strategy, it will identify positive asymptomatic persons who are potential spreaders and transmitters of the disease;

To achieve a significant and expanded number of target populations to be tested, the Philippine Society of Pathologists Inc. (PSP Inc.) recommends the following:

1. There must be a comprehensive and cost-effective strategy in place to implement pooled testing;

2. Pooled testing shall not be done on the following:

A. Symptomatic individuals

B. Recovered (although asymptomatic) patients or retesting of previously positive individuals

C. Close contacts (household and family members) of positive individuals

3. Sample pooling is an expanded and targeted testing strategy for screening ASYMPTOMATIC PERSONS. We recommend pooled testing in the following targeted populations:
A. Low prevalence communities (10\% or less) for epidemiologic surveillance and aggressive contact tracing;
B. Targeted community testing in areas that are under lockdown to identify additional infected individuals and to guide in decisions for lifting the lockdown;
C. Surveillance of health care workers and all workers in the health care facility
D. Workplace testing to include factory workers, market vendors, call center agents, transportation workers, and others;
E. Border testing at airports and seaports for inbound foreign travelers and returning residents;
F. Overseas deployment of OFWs;
G. Returning OFWs;
H. Frontline government workers (police, military, quarantine, immigration officers to name a few);
I. Locally Stranded Individuals (LSI)
$\mathrm{J}$. Any other vulnerable populations to be determined in the future

4. The initial recommendation is to use a pool sample of 5 , until an accurate prevalence of cases with the presence of the SARS -CoV-2 virus is identified in the population.

5. Quality and accuracy of the sample pooling strategy must be enhanced and maintained thru:
A. Defined standards and procedures of practice
B. Training
C. Quality assurance
D. Monitoring 
6. There is a need to have a technical validation process for all COVID-19 testing laboratories which will participate in the pooled testing;

7. Sample pooling is a strategy to ensure a wider population to be tested in a cheaper, faster, and more efficient manner. More importantly, there is the need to implement contact tracing for those exposed to persons who tested positive for the presence of the SARS-CoV-2 virus in order to break the cycle of transmission at the earliest time possible;

8. Pooled testing will accelerate the analytical process but there is a need to address the pre-analytical and post-analytical phases when dealing with thousands of specimens to be handled the same time. The pre-analytical and post-analytical processes should ensure an organized, systematic, and streamlined set pf procedures for mass specimen collection, documentation, and reporting, as well as include the use of appropriate Information Technology systems.

9. This is a dynamic situation and PSP will be issuing updated guidelines when circumstances dictate.

In summary, the PSP Inc. stands ready to be in the forefront of diagnostic testing during this pandemic. We are ready to assist in the implementation of these recommendations in an effort to reduce transmission of SARS-CoV-2 in the country. 